\title{
A non-computational approach to the gradings on $\mathfrak{f}_{4}$
}

\author{
Cristina Draper Fontanals
}

\begin{abstract}
The fine group gradings on the exceptional Lie algebra $\mathfrak{f}_{4}$ have previously been determined by means of computational methods. A new argument is given to prove that there are just four fine gradings on $\mathfrak{f}_{4}$.
\end{abstract}

\section{Introduction}

There has been a lot of research around the gradings on simple Lie algebras during the last years. Probably one of the reasons of such activity is that fine gradings are closely related to the structure of the algebras. To be more precise, gradings on classical Lie algebras have been studied in [5], [3] and [17] and lately revised in [15] and [2] to obtain an irredundant list of nonequivalent fine gradings and nonisomorphic gradings respectively; gradings on $\mathfrak{g}_{2}$ appear in [9] and [4]; gradings on $\mathfrak{d}_{4}$ are in [12] and [15], jointly with some descriptions in [13]; and gradings on $\mathfrak{f}_{4}$ can be found in [11].

In fact, there are descriptions of fine gradings on $\mathfrak{f}_{4}$ also in [14] and [10], but these papers can not assure if the described gradings cover the whole list of fine gradings. The only proof of this fact appears in [11], and it is a computationalbased proof, quite technical, which needs a precise knowledge of the coordinate matrices of automorphisms of $\mathfrak{f}_{4}$ extending the action of elements in the Weyl group.

It does not happen only in $\mathfrak{f}_{4}$, but in general, that it is not a difficult task to describe gradings (it only requires enough knowledge of the algebra) but it could be quite difficult to prove that every fine grading is equivalent to one grading of a determined list. The classical case was the first to be studied because the authors worked with associative techniques, taking advantage that these algebras live in matrix algebras. But in the exceptional case several different techniques have been tested until now. The computational proof in the case of $\mathfrak{f}_{4}$ is based in the fact that the subgroup of automorphisms producing the grading is contained in the normalizer of a maximal torus of the automorphism group, thus the authors

Mathematics Subject Classification (2010): 17B70, 17B25.

Keywords: Fine grading, exceptional Lie algebra, $\mathfrak{f}_{4}$, MAD-group. 
worked with a precise matricial description of the elements in such normalizer (these matricial descriptions can be obtained in [25]).

Our objective in this paper is to provide an alternative proof of the fact that there are 4 fine gradings on $\mathfrak{f}_{4}$ up to equivalence. This proof will not use computational tools, but the result that the 2-groups of the automorphism group of $\mathfrak{f}_{4}$ live in $\operatorname{Spin}(9)$ and hence, after projection $(\operatorname{Spin}(9)$ is the universal covering of $\mathrm{SO}(9)$ ) inside some maximal abelian diagonalizable group of $\mathrm{SO}(9)$. But all the gradings on the Lie algebra so(9) are elementary (induced by the natural module), and can be easily extracted from [3]. Therefore, in an indirect way, we will also use matrix methods.

The purpose is to make the paper as selfcontained as possible. It is organized as follows. We will work over an algebraically closed field $\mathbb{K}$ of characteristic zero, although this hypothesis could have been relaxed. Section 2 contains the interpretation of the gradings in terms of algebraic groups, in particular of the fine gradings by means of MAD-groups. There are also several useful results about the structure of the MAD-groups of an automorphism group of a semisimple Lie algebra, applicable not only to $\mathfrak{f}_{4}$. Probably the most interesting result in this part is that every MAD-group (different from the maximal torus) contains a nontoral $p$-group for certain prime $p$, which must be 2 or 3 in the $\mathfrak{f}_{4}$-case. Afterwards we exhibit in Section 3 some natural descriptions of the four fine gradings on $\mathfrak{f}_{4}$. The objective will be to prove, in Section 6 , that these are all the fine gradings on $\mathfrak{f}_{4}$ up to equivalence. The machinery is developed in Section 4 and Section 5, devoted to 2-groups and 3-groups respectively. The key point is that if the MAD-group is not isomorphic to $\mathbb{Z}_{3}^{3}$, then it contains an order 2 automorphism fixing a subalgebra of type $\mathfrak{b}_{4}$ and hence it lives in its centralizer, which is the spin group. In order to compute the MAD-groups of Spin(9), we provide a concrete description of this spin group, then of the projections of some of its elements in the orthogonal group $\mathrm{O}(9) \cong \operatorname{aut}\left(\mathfrak{b}_{4}\right)$, which allows us to work with the MAD-groups of $\mathrm{O}(9)$. We also enclose the model of $\mathfrak{f}_{4}$ based on $\mathfrak{b}_{4}$ in order to get a precise description of the relationship between $\operatorname{aut}\left(\mathfrak{b}_{4}\right)$ and $\operatorname{aut}\left(\mathfrak{f}_{4}\right)$. A similar development, but less detailed, is done in Section 5 to extract the information about the 3 -groups from $\mathrm{SL}(3) \times{ }_{\mathbb{Z}_{3}} \mathrm{SL}(3)$.

\section{Generalities on gradings}

\subsection{Basic definitions}

Let $\mathcal{L}$ be a finite-dimensional Lie $\mathbb{K}$-algebra. The term grading will always mean group grading, that is, a decomposition in vector subspaces $\mathcal{L}=\oplus_{g \in G} \mathcal{L}_{g}$ where $G$ is a finitely generated abelian group and the homogeneous spaces verify $\left[\mathcal{L}_{g}, \mathcal{L}_{h}\right] \subset$ $\mathcal{L}_{g h}$ for any $g, h \in G$ (denoting by juxtaposition the product in $G$ ). We also assume that $G$ is generated by $\operatorname{Supp}(G):=\left\{g \in G \mid \mathcal{L}_{g} \neq 0\right\}$, called the support of the grading.

Given two gradings $\mathcal{L}=\oplus_{g \in G} U_{g}$ and $\mathcal{L}^{\prime}=\oplus_{h \in H} V_{h}$, we shall say that they are isomorphic if there are a group isomorphism $\sigma: G \rightarrow H$ and an isomorphism $\varphi: \mathcal{L} \rightarrow \mathcal{L}^{\prime}$ such that $\varphi\left(U_{g}\right)=V_{\sigma(g)}$ for any $g \in G$. The above two gradings are 
said to be equivalent if there are a bijection $\sigma: \operatorname{Supp}(G) \rightarrow \operatorname{Supp}(H)$ and an isomorphism $\varphi: \mathcal{L} \rightarrow \mathcal{L}^{\prime}$ such that $\varphi\left(U_{g}\right)=V_{\sigma(g)}$ for any $g \in \operatorname{Supp}(G)$. The first grading is a refinement of the second one if there are a surjective map $\sigma: \operatorname{Supp}(G) \rightarrow \operatorname{Supp}(H)$ and an isomorphism $\varphi: \mathcal{L} \rightarrow \mathcal{L}^{\prime}$ such that $\varphi\left(U_{g}\right) \subset V_{\sigma(g)}$ for any $g \in \operatorname{Supp}(G)$.

A grading is fine if its unique refinement is the given grading. Our objective will be to classify fine gradings on $\mathfrak{f}_{4}$ up to equivalence.

\subsection{Group techniques}

The gradings on $\mathcal{L}$ can be seen as the simultaneous diagonalizations relative to quasitori of the group of automorphisms aut $(\mathcal{L})$. If $\mathcal{L}=\oplus_{g \in G} \mathcal{L}_{g}$ is a $G$-grading, the map $\psi: \mathfrak{X}(G)=\operatorname{hom}\left(G, \mathbb{K}^{\times}\right) \rightarrow \operatorname{aut}(\mathcal{L})$, mapping each $\alpha \in \mathfrak{X}(G)$ to the automorphism $\psi_{\alpha}: \mathcal{L} \rightarrow \mathcal{L}$ given by $\mathcal{L}_{g} \ni x \mapsto \psi_{\alpha}(x):=\alpha(g) x$, is a group homomorphism. Since $G$ is finitely generated, then $\psi(\mathfrak{X}(G))$ is a quasitorus. And conversely, if $Q$ is a quasitorus and $\psi: Q \rightarrow \operatorname{aut}(\mathcal{L})$ is a homomorphism, $\psi(Q)$ is formed by semisimple automorphisms and we have a $\mathfrak{X}(Q)$-grading $\mathcal{L}=\oplus_{g \in \mathfrak{X}(Q)} \mathcal{L}_{g}$ given by $\mathcal{L}_{g}=\{x \in V \mid \psi(q)(x)=g(q) x \forall q \in Q\}$, with $\mathfrak{X}(Q)$ a finitely generated abelian group.

A grading is fine if and only if the quasitorus producing the grading is a maximal abelian subgroup of semisimple elements, usually called a $M A D$ ("maximal abelian diagonalizable")-group. It is convenient to observe that the number of conjugacy classes of MAD-groups of $\operatorname{aut}(\mathcal{L})$ coincides with the number of equivalence classes of fine gradings on $\mathcal{L}$.

We would like to dive a little bit in the structure of these MAD-groups, for purposes not only for this paper, but for other Lie algebras.

\subsection{Structure of a MAD-group}

We study now the MAD-groups of $\operatorname{aut}(\mathcal{L})=: G$, for $\mathcal{L}$ a finite-dimensional semisimple Lie $\mathbb{K}$-algebra. Of course there is always at least one MAD-group, the maximal torus formed by the automorphisms fixing a Cartan subalgebra (all the maximal tori are conjugated). Any other MAD-group $Q$ has to be nontoral (that is, not contained in a torus). Moreover, as any quasitorus, this $Q$ is the direct product of a torus by a finite subgroup of $G$. The purpose of this section is to prove that $Q$ contains a nontoral $p$-group for some prime $p$, that is, if we write the finite subgroup as a direct product of $p_{i}$-groups for different primes $p_{i}$, some of the factors are nontoral. We have the conjecture that all the factors are nontoral.

Recall first a pair of facts which help to check torality. We enclose the proofs for the seek of completeness.

Lemma 2.1 (Theorem 8.2.(3) in [1]). Let $\mathcal{G}$ be a linear algebraic group over an algebraically closed field. Assume that $\mathcal{G}$ is a connected reductive group such that its commutator subgroup is simply connected. If $Q$ is a subquasitorus of $\mathcal{G}$ generated by at most two elements, then $Q$ is toral. 
Proof. Take $Q=\overline{\left\langle f_{1}, f_{2}\right\rangle}$, and consider $Z$ the centralizer of $f_{1}$ in $\mathcal{G}$, which is connected by Theorem 3.5.6, page 93, of [7]. As any semisimple element in a connected group belongs to a torus, there is a maximal torus $T$ of $Z$ such that $f_{2} \in Z$. But $f_{1}$ is in the center of $Z$ and hence in all the maximal tori of $Z$, so that $\left\langle f_{1}, f_{2}\right\rangle \subset T$ and $Q \subset T$.

Lemma 2.2 (Lemma 2 in [12]). If $T$ is a torus of $G$ and $H$ is a toral subgroup of $G$ commuting with $T$, then $H T$ is toral.

Proof. Let $Z$ be the centralizer of $H$ in $\operatorname{aut}(\mathcal{L})=G$. As $H$ is toral, there is a maximal torus $T^{\prime}$ of $\operatorname{aut}(\mathcal{L})$ such that $H \subset T^{\prime}$. Hence $T^{\prime} \subset Z$ and it is also a maximal torus of $Z$. But $T \subset Z$ so that there is $p \in Z$ such that $p T p^{-1} \subset T^{\prime}$. Consequently $p(H T) p^{-1}=H p T p^{-1} \subset H T^{\prime} \subset\left(T^{\prime}\right)^{2} \subset T^{\prime}$ and $H T$ is contained in the torus $p^{-1} T^{\prime} p$.

It is very useful to recall the version in [26] (Theorem 3.15, page 92) of the Borel-Serre Theorem, which in particular implies that every quasitorus of $G$ is contained in the normalizer of some maximal torus. But we will need a slightly improved version of this result (which also generalizes Proposition 7 of [11]).

Lemma 2.3. If $\mathrm{H}_{1}$ is a toral subgroup of $G$ and $\mathrm{H}_{2}$ is a diagonalizable subgroup of $G$ which commutes with $H_{1}$, then there is a maximal torus $T$ of $G$ such that $H_{1} \subset T$ and $H_{2}$ is contained in the normalizer $\mathrm{N}(T)$.

Proof. Let $Z=\operatorname{Cent}_{G}\left(H_{1}\right)$. As $H_{1}$ is toral, there is a torus $T_{1}$ of $G$ such that $H_{1} \subset T_{1} \subset Z$. As $T_{1}$ is connected and it contains $1_{G}$, then $T_{1} \subset Z_{0}$, where $Z_{0}$ denotes the connected component of $Z$ containing the unit. Now we apply the previously cited theorem (Theorem 3.15, page 92, of [26]) to $\mathrm{H}_{2}$, a diagonalizable subgroup of $Z$, so that there is a maximal torus $T$ of $Z$ such that $H_{2} \subset N(T)$. Note also that $H_{1} \subset T$ because $H_{1}$ is in the center of $Z_{0}$, and precisely the set of semisimple elements of $Z_{0}$ coincides with the intersection of all the maximal tori of $Z_{0}$ (one of them is our $T$ ), according to Corollary 11.1 of [6].

Lemma 2.4. If a prime $p$ does not divide the order of the Weyl group of $\mathcal{L}$, then every abelian p-group $H \leq G$ is toral.

Proof. The elements in $H$ have order a power of $p$, so that they are semisimple and, as in the previous lemma, there is a maximal torus $T$ such that $H \subset \mathrm{N}(T)$. Let us check that any $f \in H$ verifies that $f \in T$. Let us take $\pi: \mathrm{N}(T) \rightarrow \mathrm{N}(T) / T$ the projection onto the semidirect product of the Weyl group and the group of diagram automorphisms. The order of $\pi(f)$ must be a divisor of the order of $f$, certain $p^{k}$ for some $k \in \mathbb{N}$. But also the order of $\pi(f)$ divides the order of the Weyl group, which is coprime to $p^{k}$. So $\pi(f)=1$ and $f \in T$.

We will use a pair of times the following trivial result.

Lemma 2.5. If $T$ is a torus and $H_{1}$ and $H_{2}$ are finite groups of coprime orders such that $H_{2}$ commutes with $T$ and $H_{1} \subset T \times H_{2}$, then $H_{1}$ is contained in $T$.

Proof. It is clear, since the projection of $H_{1}$ in $H_{2}$ must be trivial. 
Lemma 2.6. If $T$ is a maximal torus of $G$, and $f \in \mathrm{N}(T)$ is an element of order $r \in \mathbb{N}$, then the set $T^{\langle f\rangle}$ of the elements in $T$ commuting with $f$ is equal to $S H$ for some subtorus $S$ of $T$ and a subgroup $H \subset\left\{t \in T \mid t^{r}=1_{G}\right\}$ such that $S \cap H=\left\{1_{G}\right\}$.

Proof. Recall that we have an action $\mathrm{N}(T) \times T \rightarrow T,(g, t) \mapsto g \cdot t:=g t g^{-1}$. Hence we can write $T^{\langle f\rangle}=\{t \in T \mid f t=t f\}=\{t \in T \mid f \cdot t=t\}$. As it is a diagonalizable group (a quasitorus), there are a subtorus $S$ and a finite group $H$ such that $T^{\langle f\rangle}=S H$ and $S \cap H=\left\{1_{G}\right\}$.

Note that the map $s: T \rightarrow T$ given by $s(t)=\pi_{i=0}^{r-1} f^{i} \cdot t$ is an algebraic group homomorphism, so that $s(T)$ is a subtorus of $T$. As $t(f \cdot s(t))=\pi_{i=0}^{r} f^{i} \cdot t=$ $s(t)\left(f^{r} \cdot t\right)$ and $f^{r}=1_{G}$, we get that $s(t) \in T^{\langle f\rangle}$. Hence the torus $s(T)$ must be contained in the only maximal torus of $T^{\langle f\rangle}$, that is, $S$. Let us check now that if $t \in H$ then $t^{r}=1_{G}$. Indeed, as $f \cdot t=t$, we have $s(t)=t^{r}$, so that $t^{r} \in H \cap s(T) \subset H \cap S=\left\{1_{G}\right\}$.

Lemma 2.7. If $H_{1}$ and $H_{2}$ are toral subgroups of $G$ which commute, of coprime orders $r$ and $s$ respectively, then the group $\mathrm{H}_{1} \mathrm{H}_{2}$ is toral.

Proof. As in Lemma 2.3, there is a torus $T$ such that $H_{1} \subset T$ and $H_{2} \subset \mathrm{N}(T)$. We can take $H_{2}=\left\langle\left\{f_{1}, \ldots, f_{m}\right\}\right\rangle$ for certain generators $f_{i}$. Call $s_{i}$ the order of $f_{i}$, which is a divisor of $s$. By Lemma 2.6 and the notations therein, $H_{1} \subset T^{\left\langle f_{1}\right\rangle}=$ $\left\{t \in T \mid t f_{1}=f_{1} t\right\}$ and it coincides with $T_{1} V_{1}$ for some $T_{1}$ subtorus of $T$ and a subgroup $V_{1} \subset\left\{t \in T \mid t^{s_{1}}=1\right\}$, with $T_{1} \cap V_{1}=\{1\}$. As the cardinal of $V_{1}$ divides $s_{1} \operatorname{dim} T$, it also divides $s^{\operatorname{dim} T}$ and hence this cardinal is coprime to $r$ (recall that $\operatorname{gcd}(\mathrm{r}, \mathrm{s})=1)$. Then, by applying Lemma 2.5 , we get that $H_{1} \subset T_{1}$. Now $T_{1}$ is a torus and $\left\langle\left\{f_{1}\right\}\right\rangle$ is toral (it is contained in $H_{2}$ ) commuting with $T_{1}$, so, by Lemma 2.2 we get that $T_{1}\left\langle\left\{f_{1}\right\}\right\rangle$ is toral and hence $H_{1}\left\langle\left\{f_{1}\right\}\right\rangle$ is toral too. Now the process begins again. By Lemma 2.3, there is a torus $T^{\prime}$ such that $H_{1} \cup\left\{f_{1}\right\} \subset T^{\prime}$ and $H_{2} \subset \mathrm{N}\left(T^{\prime}\right)$. Hence $H_{1} \cup\left\{f_{1}\right\} \subset T^{\left\langle f_{2}\right\rangle}=\left\{t \in T \mid t f_{2}=f_{2} t\right\}$, which, according to Lemma 2.6, coincides with $T_{2} V_{2}$ for some subtorus $T_{2}$ of $T^{\prime}$ and a subgroup $V_{2} \subset\left\{t \in T \mid t^{s_{2}}=1\right\}$ such that $T_{2} \cap V_{2}=\{1\}$. Taking into account that the order of $H_{1}$ is $r$, coprime to the cardinal of $V_{2}$ (which is a divisor of a power of $s$ ), we can apply Lemma 2.5 to conclude that $H_{1} \subset T_{2}$. We get that $\left\langle T_{2}, f_{1}, f_{2}\right\rangle$ is toral by applying Lemma 2.2 to the torus $T_{2}$ and to the toral subgroup $\left\langle f_{1}, f_{2}\right\rangle$, which commutes with $T_{2}$. As $\left\langle H_{1}, f_{1}, f_{2}\right\rangle$ is contained in $\left\langle T_{2}, f_{1}, f_{2}\right\rangle$, it is also toral. The application of lemmas 2.3, 2.6, 2.5 and 2.2 allows to conclude the torality of $\left\langle H_{1} \cup\left\{f_{j} \mid j=1, \ldots, i\right\}\right\rangle$ from the one of $\left\langle H_{1} \cup\left\{f_{j} \mid j=1, \ldots, i-1\right\}\right\rangle$, so an induction argument ends the proof.

Hence,

Corollary 2.8. If $H_{i}$ is a finite toral $p_{i}$-subgroup of $G$ for each $i \in\{1, \ldots, s\}$, with $p_{i}$ prime and $p_{i} \neq p_{j}$ if $i \neq j$, and the group generated by $H_{1} \cup \cdots \cup H_{s}$ is abelian, then such group is toral.

Some immediate consequences are the following, for general Lie algebras and for our concrete case: 
Corollary 2.9. Any nontoral quasitorus of $G$ contains a nontoral finite p-group for some prime $p$.

Proof. Take into account that such quasitorus is a direct product $T \times H_{1} \times \cdots \times H_{s}$ of a torus $T$ and some finite abelian $p_{i}$-groups $H_{i}$ ( $p_{i}$ prime) such that $p_{i} \neq p_{j}$ if $i \neq j$. Now apply Lemma 2.2 and the previous corollary.

Remark 2.10. We could think that every nontoral quasitorus of $G$ contains a nontoral elementary $p$-group for some prime $p$. This result would be relevant for the study of the gradings on the remaining exceptional Lie algebras (type $\mathfrak{e}$ ), because there is a lot of information about elementary $p$-groups (the maximal ones are detailed in [16] and for $p=3$ in [1]). But that conjecture is not true: take, for instance, the quasitorus $Q=\left\langle\left\{t_{-1,1,-1,1}, t_{1,-1,-1,1}, \widetilde{\sigma}_{105} t_{1,1,1, i}\right\}\right\rangle \cong \mathbb{Z}_{2}^{2} \times \mathbb{Z}_{4}$ (notations as in [11]). It is nontoral, but every proper subquasitorus is toral, in particular that one isomorphic to $\mathbb{Z}_{2}^{3}$.

Corollary 2.11. Any abelian p-subgroup of $\operatorname{aut}\left(\mathfrak{f}_{4}\right)$ is toral if $p>3$. Any nontoral quasitorus of $\operatorname{aut}\left(\mathfrak{f}_{4}\right)$ contains either a finite nontoral 2-group or a finite nontoral 3-group.

Proof. It is a consequence of Corollary 2.9 and Lemma 2.4, because the cardinal of the Weyl group is $1152=2^{7} 3^{2}$, with 2 and 3 the only prime divisors.

We will need to precise a little more for the $\mathfrak{f}_{4}$-case. Although we have not achieved to prove that any quasitorus of $\operatorname{aut}(\mathcal{L})$ is product of a torus times several $p_{i}$-nontoral groups, what is true is the next result.

Proposition 2.12. If $Q=T \times P \times R$ is a MAD-group of $G=\operatorname{aut}(\mathcal{L})$, for $\mathcal{L}$ a finite-dimensional semisimple Lie algebra, with $T$ a torus, $R$ a finite nontoral p-group ( $p$ prime) and $P$ a nontrivial toral group of order coprime to $p$, then $R$ contains a proper nontoral subquasitorus.

Proof. Take $R^{\prime}$ a maximal toral subquasitorus of $R$. By Lemma 2.7 and Lemma 2.2, the subquasitorus $T \times P \times R^{\prime}$ of $Q$ is also toral, and according to Lemma 2.3, there is a maximal torus $T^{\prime}$ of $G$ such that $T \times P \times R^{\prime} \subset T^{\prime}$ and $R=\left\langle R^{\prime} \dot{\cup}\left\{f_{1}, \ldots, f_{r}\right\}\right\rangle \subset$ $\mathrm{N}\left(T^{\prime}\right)$ with $\left\langle R^{\prime} \cup\left\{f_{1}, \ldots, f_{i}\right\}\right\rangle \subsetneq\left\langle R^{\prime} \cup\left\{f_{1}, \ldots, f_{i+1}\right\}\right\rangle$ for all $i=1, \ldots, r-1$. Note that the quasitorus generated by $R^{\prime} \cup\left\{f_{i}\right\}$ is nontoral for all $i=1, \ldots, r$. We have only to prove that $r \geq 2$. But if $r=1$, the maximality of $Q$ implies that $\left(T^{\prime}\right)^{\left\langle f_{1}\right\rangle}=T \times P \times R^{\prime}$, a contradiction with Lemma 2.6.

Corollary 2.13. Any MAD-group of aut $\left(\mathfrak{f}_{4}\right)$ which does not contain a nontoral 3 -group is $T \times R_{2} \times R$, where $T$ is a torus, $R$ is a finite toral group of odd order and $R_{2}$ is a finite nontoral 2-group, and either $R$ is trivial or $R_{2}$ has at least four direct factors.

Proof. It is enough to apply the previous proposition jointly with Corollary 2.11 and Lemma 2.1, since aut $\left(\mathfrak{f}_{4}\right)$ is simply connected.

In the last section we will prove that the group $R$ in Corollary 2.13 has necessarily to be trivial. 


\section{Description of gradings on $\mathfrak{f}_{4}$}

There are four fine gradings on $\mathfrak{f}_{4}$ described in [11] and in [10]. We enclose here a description of each of them for the seek of completeness, since our main aim is to prove that they are essentially all the possible fine gradings on $\mathfrak{f}_{4}$. These descriptions would also work for arbitrary (algebraically closed) fields of characteristic different from 2 or 3 . All the gradings on the symmetric composition algebras, as well as the different constructions used for $\mathfrak{f}_{4}$, can be found in detail in Sections 4 and 5 of $[14]$.

Given a symmetric composition algebra $(C, *, b)$ of dimension 8 , consider the orthogonal Lie algebra

$$
\mathrm{o}(C, b)=\left\{d \in \operatorname{End}_{\mathbb{K}}(C) \mid b(d(x), y)+b(x, d(y))=0 \forall x, y \in C\right\},
$$

and the subalgebra of $\mathrm{o}(C, b)^{3}$ (with componentwise multiplication) defined by

$$
\mathfrak{t r i}(C, *, b)=\left\{\left(d_{0}, d_{1}, d_{2}\right) \in \mathrm{o}(C, b)^{3} \mid d_{0}(x * y)=d_{1}(x) * y+x * d_{2}(y) \forall x, y \in C\right\},
$$

called the triality algebra. One can form the $\mathbb{Z}_{2}^{2}$-graded Lie algebra

$$
\mathcal{L}=\operatorname{tri}(C, *, b) \oplus \iota_{0}(C) \oplus \iota_{1}(C) \oplus \iota_{2}(C),
$$

where the bracket is given by

- $\operatorname{tri}(C, *, b)$ is a Lie subalgebra of $\mathcal{L}$,

- $\left[\left(d_{0}, d_{1}, d_{2}\right), \iota_{i}(x)\right]=\iota_{i}\left(d_{i}(x)\right)$,

- $\left[\iota_{i}(x), \iota_{i+1}(y)\right]=\iota_{i+2}(x * y)$ (indices modulo 3$)$,

- $\left[\iota_{i}(x), \iota_{i}(y)\right]=\theta^{i}\left(t_{x, y}\right)$,

being $t_{x, y}$ the element in $\operatorname{tri}(C, *, b)$ defined by

$$
t_{x, y}=\left(\sigma_{x, y}, \frac{1}{2} b(x, y) \mathrm{id}_{C}-r_{x} l_{y}, \frac{1}{2} b(x, y) \mathrm{id}_{C}-l_{x} r_{y}\right)
$$

with $\sigma_{x, y}(z)=b(x, z) y-b(y, z) x, r_{x}(z)=z * x$ and $l_{x}(z)=x * z$ for all $x, y, z \in C$; and where $\theta$ denotes the order 3 automorphism of $\operatorname{tri}(C, *, b)$ given by $\theta\left(d_{0}, d_{1}, d_{2}\right):=$ $\left(d_{2}, d_{0}, d_{1}\right)$. This algebra is of type $\mathfrak{f}_{4}$ independently of the considered 8-dimensional symmetric composition algebra $C$. There are two of such algebras up to isomorphism: the Okubo algebra $\mathrm{Ok}$ and the para-Hurwitz algebra $\mathrm{pH}$. The algebra Ok has a natural $\mathbb{Z}_{3}^{2}$-grading (coming from the nontoral $\mathbb{Z}_{3}^{2}$-grading on the matrix algebra $\left.\operatorname{Mat}_{3 \times 3}(\mathbb{K})\right)$ and the algebra $\mathrm{pH}$ has a natural $\mathbb{Z}_{2}^{3}$-grading (coming from the $\mathbb{Z}_{2}^{3}$-grading on the octonion algebra). So, we can consider on $\mathcal{L} \cong \mathfrak{f}_{4}$ :

A $\mathbb{Z}^{4}$-grading given by the root decomposition on $\mathcal{L}$ relative to a Cartan subalgebra.

A $\mathbb{Z}_{3}^{3}$-grading obtained by combining the $\mathbb{Z}_{3}^{2}$-grading on Ok with the $\mathbb{Z}_{3}$-grading on $\mathcal{L}$ induced by the triality automorphism $\theta$. 
A $\mathbb{Z}_{2}^{5}$-grading obtained by combining the $\mathbb{Z}_{2}^{3}$-grading on $\mathrm{pH}$ with the following $\mathbb{Z}_{2}^{2}$-grading on $\mathcal{L}: \mathcal{L}_{(\overline{0}, \overline{0})}=\operatorname{tri}(C, *, b), \mathcal{L}_{(\overline{0}, \overline{1})}=\iota_{0}(C), \mathcal{L}_{(\overline{1}, \overline{0})}=\iota_{1}(C)$ and $\mathcal{L}_{(\overline{1}, \overline{1})}=\iota_{2}(C)$.

A $\mathbb{Z}_{2}^{3} \times \mathbb{Z}$-grading. Consider the Albert algebra $\mathcal{J}=\mathbb{K}^{3} \oplus \iota_{0}(C) \oplus \iota_{1}(C) \oplus \iota_{2}(C)$ with the product described in Theorem 5.15 of [14], where $C$ is the paraHurwitz algebra $\mathrm{pH}$. Such algebra $\mathcal{J}$ has a $\mathbb{Z}$-grading produced as the simultaneous diagonalization relative to $2\left[r_{\iota_{0}(1)}, r_{(1,0,0)}\right] \in \operatorname{Der}(\mathcal{J})\left(r_{x}\right.$ denotes the multiplication operator in $\mathcal{J})$. It is compatible with the $\mathbb{Z}_{2}^{3}$-grading on $\mathrm{pH}$, and it induces the corresponding grading on $\operatorname{Der}(\mathcal{J}) \cong \mathfrak{f}_{4}$.

\section{2-groups of $\operatorname{aut}\left(\mathfrak{f}_{4}\right)$}

Taking in mind Corollary 2.13, our programme will be: First we will try to obtain all the information about the 2-groups of aut $\left(\mathfrak{f}_{4}\right)$ by means of the spin group, and afterwards we will extract the information about the 3-groups from the special linear groups.

\subsection{Spin group}

Let $V$ be a 9-dimensional $\mathbb{K}$-vector space endowed with a nondegenerate quadratic form $q: V \rightarrow \mathbb{K}$. Let $b_{q}: V \times V \rightarrow \mathbb{K}$ be the associated symmetric bilinear form given by $b_{q}(x, y)=\frac{1}{2}(q(x+y)-q(x)-q(y))$. Recall that the orthogonal group is $\mathrm{O}(V, q)=\left\{f \in \operatorname{gl}(V) \mid b_{q}(x, y)=b_{q}(f(x), f(y)) \forall x, y \in V\right\}$ and the special orthogonal group is $\mathrm{SO}(V, q)=\{f \in \mathrm{O}(V, q) \mid \operatorname{det}(f)=1\}$.

It is well known that the spin group is the universal covering of the special orthogonal group. A treatment of spin groups valid for our context can be found in Chapter IV of [23]. Let us concrete a description suitable for our purposes.

Let $T(V)=\sum_{n=0}^{\infty} V^{\otimes n}$ be the associative tensor algebra. Let $I$ be the ideal of $T(V)$ generated by $\{v \otimes v-q(v) 1 \mid v \in V\}$. The Clifford algebra is the (unital) associative algebra given by the quotient

$$
\mathrm{Cl}(V, q)=T(V) / I
$$

and $\mathrm{Cl}(V, q)^{-}$is, as always, the same vector space endowed with the bracket $[x, y]=$ $x y-y x$. Let

$$
\mu: \mathrm{Cl}(V, q) \rightarrow \mathrm{Cl}(V, q)
$$

be the automorphism which extends $\mu(v)=-v$ for $v \in V$. As $\mu$ is an order 2 automorphism, it induces a $\mathbb{Z}_{2}$-grading on the Clifford algebra, with even and odd parts denoted respectively by $\mathrm{Cl}(V, q)_{\overline{0}}$ and $\mathrm{Cl}(V, q)_{\overline{1}}$. If we denote by $\mathrm{Cl}(V, q)^{\times}$the group of invertible elements in the Clifford algebra, the Clifford group is defined by

$$
\Gamma(V, q):=\left\{x \in \mathrm{Cl}(V, q)^{\times} \mid \mu(x) V x^{-1} \subset V\right\} .
$$

Obviously we can consider the group homomorphism

$$
\begin{aligned}
\rho: \Gamma(V, q) & \rightarrow \mathrm{GL}(V) \\
x & \mapsto \rho(x) ; \quad \rho(x)(v)=\mu(x) v x^{-1} \forall v \in V .
\end{aligned}
$$


As $q\left(\mu(x) v x^{-1}\right)=q(v)$ for any $v \in V$, we actually have a representation $\rho: \Gamma(V, q)$ $\rightarrow \mathrm{O}(V, q)$. Any $v \in V$ such that $q(v) \neq 0$ is invertible, and $-v w v^{-1}=(w v-$ $\left.2 b_{q}(v, w) 1\right) v^{-1}=w-2 b_{q}(v, w) / b_{q}(v, v) v$, hence $v \in \Gamma(V, q)$ and $\rho(v)$ is the reflection relative to the hyperplane orthogonal to $v$. According to the CartanDieudonné Theorem, every isometry of $V$ is composition of reflections relative to hyperplanes orthogonal to nonisotropic vectors, so that $\rho(\Gamma(V, q))=\mathrm{O}(V, q)$, $\Gamma(V, q)=\left\{\lambda u_{1} \ldots u_{r} \mid \lambda \in \mathbb{K}^{\times}, u_{i} \in V, q\left(u_{i}\right) \neq 0, r \geq 0\right\}$ and $\operatorname{ker}(\rho)=\mathbb{K}^{\times}$ $(=\mathbb{K} \backslash\{0\})$. As $\operatorname{det}(\rho(v))=-1$, we also conclude that $\rho\left(\Gamma(V, q) \cap \mathrm{Cl}(V, q)_{\overline{0}}\right)=$ $\mathrm{SO}(V, q)$ and $\Gamma(V, q) \cap \mathrm{Cl}(V, q)_{\overline{0}}=\left\{\lambda u_{1} \ldots u_{2 r} \mid \lambda \in \mathbb{K}^{\times}, u_{i} \in V, q\left(u_{i}\right) \neq 0, r \geq 0\right\}$. Hence we have the following short exact sequences:

$$
\begin{aligned}
& 1 \rightarrow \mathbb{K}^{\times} \rightarrow \Gamma(V, q) \rightarrow \mathrm{O}(V, q) \rightarrow 1 \\
& 1 \rightarrow \mathbb{K}^{\times} \rightarrow \Gamma(V, q) \cap \mathrm{Cl}(V, q)_{\overline{0}} \rightarrow \mathrm{SO}(V, q) \rightarrow 1 .
\end{aligned}
$$

The spin group lives inside the even part of the Clifford group. Take the spinor norm $N: \Gamma(V, q) \rightarrow \mathbb{K}^{\times}$given by $N(x)=\mu\left(x^{*}\right) x$, where $*$ is the involution given by $v^{*}=v$ for any $v \in V$. In particular, $N(v)=-q(v)$. The spin group is defined as $\operatorname{Spin}(V, q)=\left\{x \in \Gamma(V, q) \cap \mathrm{Cl}(V, q)_{\overline{0}} \mid N(x)=1\right\}$. As $N\left(\lambda u_{1} \ldots u_{2 r}\right)=\lambda^{2} \pi_{i=1}^{2 r} q\left(u_{i}\right)$ and $\mathbb{K}$ is algebraically closed, we can scale to get

$$
\operatorname{Spin}(V, q)=\left\{ \pm \pi_{i=1}^{2 r} u_{i} \mid u_{i} \in V, q\left(u_{i}\right)=1\right\}
$$

and now it is clear that $\left.\rho\right|_{\operatorname{Spin}(V, q)}: \operatorname{Spin}(V, q) \rightarrow \operatorname{SO}(V, q)$ is still an epimorphism, with kernel $\{ \pm 1\} \cong \mathbb{Z}_{2}$. From now on $\rho$ will denote this restriction $\left.\rho\right|_{\operatorname{Spin}(V, q)}$.

\subsection{Distinguished elements in the spin group}

Let us focus our attention on some remarkable elements in the Clifford and spin groups, which will be of special relevance for our description of the MAD-groups of $\operatorname{Spin}(V, q)$. Let

$$
B:=\left\{e_{0}, u_{1}, u_{2}, u_{3}, u_{4}, v_{1}, v_{2}, v_{3}, v_{4}\right\}
$$

be a $\mathbb{K}$-basis of $V$ such that the matrix of $b_{q}$ relative to $B$ is $\left(\begin{array}{ccc}1 & 0 & 0 \\ 0 & 0 & I_{4} \\ 0 & I_{4} & 0\end{array}\right)$. We denote also by

$$
\begin{array}{llll}
e_{1}=\frac{1}{\sqrt{2}}\left(u_{1}+v_{1}\right), & e_{3}=\frac{1}{\sqrt{2}}\left(u_{2}+v_{2}\right), & e_{5}=\frac{1}{\sqrt{2}}\left(u_{3}+v_{3}\right), & e_{7}=\frac{1}{\sqrt{2}}\left(u_{4}+v_{4}\right), \\
e_{2}=\frac{i}{\sqrt{2}}\left(u_{1}-v_{1}\right), & e_{4}=\frac{i}{\sqrt{2}}\left(u_{2}-v_{2}\right), & e_{6}=\frac{i}{\sqrt{2}}\left(u_{3}-v_{3}\right), & e_{8}=\frac{i}{\sqrt{2}}\left(u_{4}-v_{4}\right),
\end{array}
$$

where $\mathfrak{i} \in \mathbb{K}$ is a primitive fourth root of the unit $\left(\mathfrak{i}^{2}=-1\right)$. Thus, the matrix of $b_{q}$ relative to the basis

$$
B^{\prime}:=\left\{e_{0}, e_{1}, e_{2}, e_{3}, e_{4}, e_{5}, e_{6}, e_{7}, e_{8}\right\}
$$

is the identity matrix $I_{9}$. Observe first that $q\left(\frac{1}{\sqrt{2}}\left(\beta u_{i}+\frac{1}{\beta} v_{i}\right)\right)=1$ for any $\beta \in \mathbb{K}^{\times}$, $i=1,2,3,4\left(\left\{e_{1}, \ldots, e_{8}\right\}\right.$ are particular cases $)$. If we denote by $[f]_{B^{\prime}}$ the matrix associated to $f \in \mathrm{O}(V, q)$ with respect to the base $B^{\prime}$, when computing the matrix related to $\rho\left(\frac{1}{\sqrt{2}}\left(\beta u_{i}+\frac{1}{\beta} v_{i}\right)\right)$, the block corresponding to $\left\{e_{2 i-1}, e_{2 i}\right\} \subset B^{\prime}$ is

$$
R_{\beta}:=\frac{1}{2}\left(\begin{array}{cc}
-\beta^{2}-\frac{1}{\beta^{2}} & \mathfrak{i}\left(\beta^{2}-\frac{1}{\beta^{2}}\right) \\
\mathfrak{i}\left(\beta^{2}-\frac{1}{\beta^{2}}\right) & \beta^{2}+\frac{1}{\beta^{2}}
\end{array}\right) .
$$


Hence the matrix related to the image of

$$
\left(\frac{1}{\sqrt{2}}\left(\beta u_{i}+\frac{1}{\beta} v_{i}\right)\right)\left(\frac{1}{\sqrt{2}}\left(u_{i}+v_{i}\right)\right)=\frac{1}{2}\left(\beta u_{i} v_{i}+\frac{1}{\beta} v_{i} u_{i}\right) \in \operatorname{Spin}(V, q)
$$

has a block of the form

$$
S_{\beta}:=R_{\beta} R_{1}=\frac{1}{2}\left(\begin{array}{cc}
\beta^{2}+\frac{1}{\beta^{2}} & \mathfrak{i}\left(\beta^{2}-\frac{1}{\beta^{2}}\right) \\
-\mathfrak{i}\left(\beta^{2}-\frac{1}{\beta^{2}}\right) & \beta^{2}+\frac{1}{\beta^{2}}
\end{array}\right) .
$$

Thus the element

$s_{\alpha \beta \delta \epsilon}:=\frac{1}{16}\left(\alpha u_{1} v_{1}+\frac{1}{\alpha} v_{1} u_{1}\right)\left(\beta u_{2} v_{2}+\frac{1}{\beta} v_{2} u_{2}\right)\left(\delta u_{3} v_{3}+\frac{1}{\delta} v_{3} u_{3}\right)\left(\epsilon u_{4} v_{4}+\frac{1}{\epsilon} v_{4} u_{4}\right)$

belongs to $\operatorname{Spin}(V, q)$ and $\left[\rho\left(s_{\alpha \beta \delta \epsilon}\right)\right]_{B^{\prime}}=\left(\begin{array}{ccccc}1 & 0 & 0 & 0 & 0 \\ 0 & S_{\alpha} & 0 & 0 & 0 \\ 0 & 0 & S_{\beta} & 0 & 0 \\ 0 & 0 & 0 & S_{\delta} & 0 \\ 0 & 0 & 0 & 0 & S_{\epsilon}\end{array}\right)$. Moreover,

$$
\mathcal{T}=\left\{s_{\alpha \beta \delta \epsilon} \mid \alpha, \beta, \delta, \epsilon \in \mathbb{K}^{\times}\right\}
$$

is a torus of $\operatorname{Spin}(V, q)$, since $s_{\alpha \beta \delta \epsilon} s_{\alpha^{\prime} \beta^{\prime} \delta^{\prime} \epsilon^{\prime}}=s_{\alpha \alpha^{\prime} \beta \beta^{\prime} \delta \delta^{\prime} \epsilon \epsilon^{\prime}}$.

On the other hand, $\rho\left(e_{i}\right)\left(e_{j}\right)=(-1)^{\delta_{i j}} e_{j}$ for $\delta_{i j}$ the Kronecker symbol, so that

$$
d_{i}:=\left[\rho\left(e_{i}\right)\right]_{B^{\prime}}=\operatorname{diag}\left\{(-1)^{\delta_{i j}}\right\}_{j=0, \ldots, 8}
$$

is the diagonal matrix of size 9 whose entries in the diagonal are all 1's up to one -1 in the $i$ th position. Hence $e_{i} e_{j} \in \operatorname{Spin}(V, q)$ and $\left[\rho\left(e_{i} e_{j}\right)\right]_{B^{\prime}}=d_{i} d_{j}$.

\subsection{Model of $\mathfrak{f}_{4}$ based on $\mathfrak{b}_{4}$}

We describe in this subsection the $\mathbb{Z}_{2}$-grading on $\mathfrak{f}_{4}$ such that $\operatorname{Spin}(V, q)$ is precisely the subgroup of automorphisms preserving the grading. This kind of gradings on $\mathfrak{f}_{4}$ whose even part type is $\mathfrak{b}_{4}$ is well known, appearing for instance in [19].

With the notations of subsections 4.1 and 4.2 , the orthogonal algebra

$$
\operatorname{so}(V, q)=\left\{f \in \operatorname{gl}(V) \mid b_{q}(f(x), y)+b_{q}(x, f(y))=0 \forall x, y \in V\right\}
$$

is a Lie algebra of type $\mathfrak{b}_{4}$. The space $W=\operatorname{span}\left\langle u_{1}, \ldots, u_{4}\right\rangle$ is a totally isotropic subspace of $V$. Consider the exterior algebra

$$
S:=\wedge W=\mathbb{K} \oplus W \oplus \wedge^{2}(W) \oplus \wedge^{3}(W) \oplus \wedge^{4}(W)
$$

with the $\mathbb{Z}$-grading given by $|x|=n$ if $x \in \wedge^{n}(W)$. Thus $\operatorname{End}(S)=: E=\oplus_{n \in \mathbb{Z}} E_{n}$ is also $\mathbb{Z}$-graded, for $E_{n}=\left\{f \in \operatorname{End}(S) \mid f\left(\wedge^{m}(W)\right) \subset \wedge^{m+n}(W) \forall m \in \mathbb{N}\right\}$. Let us recall how $\operatorname{so}(V, q)$ acts on the 16 -dimensional vector space $S$, following $§ 8$. A of [24]. First consider the map

$$
\gamma: V \rightarrow \operatorname{End}(\wedge W)
$$

given by

$$
\gamma\left(\lambda e_{0}+u+v\right)=\lambda \tilde{I}+l_{u}+d_{v}
$$

where $u \in W, v \in \operatorname{span}\left\langle v_{1}, \ldots, v_{4}\right\rangle$ (which can be identified to $W^{*}$ by means of $\left.v \mapsto b_{q}(v,-)\right)$, and 
- $\tilde{I} \in E_{0}$ is the map producing the $\mathbb{Z}_{2}$-grading on $S$, that is, $\left.\tilde{I}\right|_{\mathbb{K} \oplus \wedge^{2}(W) \oplus \wedge^{4}(W)}$ $=\mathrm{id}$ and $\left.\tilde{I}\right|_{W \oplus \wedge^{3}(W)}=-\mathrm{id}$.

- The map $l_{u}: \wedge W \rightarrow \wedge W$ is given by $l_{u}(w)=u \wedge w$ if $w \in S$. Thus $l_{u} \in E_{1}$.

- The map $d_{v}$ is defined on $\wedge^{n}(W)$ by induction on the degree $n$ : $d_{v}(1)=0$, $d_{v}(w)=2 b_{q}(v, w) 1$ for $w \in W$ and $d_{v}(x \wedge y)=d_{v}(x) \wedge y+(-1)^{|x|} x \wedge d_{v}(y)$ if $x, y \in \cup_{m=0}^{4} \wedge^{m}(W)$. In particular $d_{v} \in E_{-1}$.

It is clear that $\gamma(x)^{2}=q(x) \operatorname{id}_{\wedge W}$ for any $x \in V$, so that $\gamma$ induces a homomorphism of associative algebras $\tilde{\gamma}$ from $\mathrm{Cl}(V, q)$ to $\operatorname{End}(\wedge W)$, and, in particular, a homomorphism of Lie algebras from $\mathrm{Cl}(V, q)^{-}$to $\operatorname{gl}(\wedge W)$ (which turns out to be an isomorphism).

As we have a monomorphism $\iota: \operatorname{so}(V, q) \rightarrow \mathrm{Cl}(V, q)^{-}$given by $b_{q}(a,-) c-$ $b_{q}(c,-) a \mapsto-\frac{1}{4}[a, c]$, the composition

$$
\tilde{\gamma} \iota: \operatorname{so}(V, q) \rightarrow \operatorname{gl}(\wedge W)
$$

provides a representation of the Lie algebra $\operatorname{so}(V, q)$. We know that this $\operatorname{so}(V, q)$ module $\wedge W$ is the spin module, that is, it is irreducible with maximal weight $\lambda_{4}$ ( $\lambda_{i}$ the fundamental weights). Indeed, $\mathfrak{h}=\left\langle h_{i} \mid i=1, \ldots, 4\right\rangle$ is a Cartan subalgebra of $\operatorname{so}(V, q)$ for $h_{i}:=b_{q}\left(v_{i},-\right) u_{i}-b_{q}\left(u_{i},-\right) v_{i}$. This element acts on $\wedge W$ as $\tilde{\gamma}\left(-\frac{1}{4}\left[v_{i}, u_{i}\right]\right)=\frac{1}{4}\left(l_{u_{i}} d_{v_{i}}-d_{v_{i}} l_{u_{i}}\right)$, in other words

$$
h_{i} \cdot\left(u_{j_{1}} \wedge \cdots \wedge u_{j_{r}}\right)= \begin{cases}\frac{1}{2} u_{j_{1}} \wedge \cdots \wedge u_{j_{r}} & \text { if } i \in\left\{j_{1}, \ldots, j_{r}\right\} \\ -\frac{1}{2} u_{j_{1}} \wedge \cdots \wedge u_{j_{r}} & \text { if } i \notin\left\{j_{1}, \ldots, j_{r}\right\} .\end{cases}
$$

Note that a set of simple roots of $\mathfrak{b}_{4}$ relative to the Cartan subalgebra $\mathfrak{h}$ is given by $\alpha_{1}(h)=\omega_{1}-\omega_{2}, \alpha_{2}(h)=\omega_{2}-\omega_{3}, \alpha_{3}(h)=\omega_{3}-\omega_{4}, \alpha_{4}(h)=\omega_{4}$, if $h=\sum_{i=1}^{4} \omega_{i} h_{i}$ is a generic element in $\mathfrak{h}$. Now a maximal vector in $\wedge W$ is $s=u_{1} \wedge u_{2} \wedge u_{3} \wedge u_{4}$, since it is annihilated by $L_{\alpha}$ for all $\alpha \in \Phi^{+}$. That means that the maximal weight $\lambda$ is given by $h \cdot s=\lambda(h) s$, so that $\lambda=\sum_{i=1}^{4} m_{i} \lambda_{i}$, where $m_{i}=h_{\alpha_{i}} \cdot s$ for $h_{\alpha_{i}}=h_{i}-h_{i+1}$ $(i \leq 3), h_{\alpha_{4}}=2 h_{4}$. Equation (4.3) gives that such maximal weight is $\lambda=\lambda_{4}$.

Now we construct

$$
\mathcal{L}=\mathcal{L}_{\overline{0}} \oplus \mathcal{L}_{\overline{1}}=\operatorname{so}(V, q) \oplus(\wedge W)
$$

with the product given by

- $\operatorname{so}(V, q)$ is a Lie subalgebra.

- If $f \in \operatorname{so}(V, q)$ and $s \in \wedge W$, we define $[f, s]=\tilde{\gamma} \iota(f)(s)$, that is, $\mathcal{L}_{\overline{0}}$ acts in $\mathcal{L}_{\overline{1}}$ by means of the spin action.

- There is, up to scalar, an unique $\operatorname{so}(V, q)$-invariant map $\wedge W \times \wedge W \rightarrow \operatorname{so}(V, q)$ (there is only one module of type $V\left(\lambda_{2}\right)$ in the decomposition into irreducible submodules of $\left.V\left(\lambda_{4}\right) \otimes V\left(\lambda_{4}\right)\right)$. To fix a scalar, we have fixed an $\operatorname{so}(V, q)$ invariant symmetric bilinear form $(\cdot \mid \cdot): \wedge W \times \wedge W \rightarrow \mathbb{K}$ (also determined up to scalar) and we have taken the dualized action of the previous one: if $s, s^{\prime} \in \wedge W$, we take $\left[s, s^{\prime}\right] \in \operatorname{so}(V, q)$ the only element satisfying $\operatorname{tr}\left(\left[s, s^{\prime}\right] f\right)=$ $\left([f, s] \mid s^{\prime}\right)$ for all $f \in \operatorname{so}(V, q)$. 
This $\mathbb{Z}_{2}$-graded Lie algebra $\mathcal{L}$ is simple of type $\mathfrak{f}_{4}$. We call $\varphi$ the grading automorphism:

$$
\left.\varphi\right|_{\mathrm{so}(V, q)}=\mathrm{id},\left.\quad \varphi\right|_{\wedge W}=-\mathrm{id} .
$$

The aim of this subsection is to prove next that the centralizer of $\varphi$ in the automorphism group of $\mathfrak{f}_{4}$ is just the group $\operatorname{Spin}(V, q)$. As usual, $\operatorname{Ad}: \operatorname{SO}(V, q) \rightarrow$ $\operatorname{gl}(\operatorname{so}(V, q))$ will denote the adjoint map given by $\operatorname{Ad} A(f)=A f A^{-1}$ for any $A \in$ $\mathrm{SO}(V, q), f \in \operatorname{so}(V, q) \equiv \mathfrak{b}_{4}$.

Proposition 4.1. If $x \in \operatorname{Spin}(V, q)$, the map $\psi_{x}: \mathfrak{f}_{4} \rightarrow \mathfrak{f}_{4}$ given by $\left.\psi_{x}\right|_{\operatorname{so}(V, q)}=$ $\operatorname{Ad} \rho(x)$ and $\left.\psi_{x}\right|_{\wedge W}=\tilde{\gamma}(x)$, is an automorphism of the Lie algebra $\mathfrak{f}_{4}$; and the map

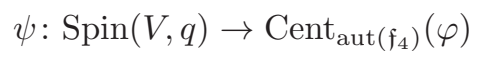

given by $x \mapsto \psi_{x}$ is a group isomorphism.

Proof. Check first that $\psi_{x}$ is an automorphism, so that $\psi$ is well defined. Take $s, s^{\prime} \in S$ and $f=b_{q}(a,-) b-b_{q}(b,-) a \in \mathfrak{b}_{4}$, for $a, b \in V$. As $[f, s]=\tilde{\gamma} \iota(f)(s)=$ $\tilde{\gamma}\left(-\frac{1}{4}[a, b]\right)(s)$, then $\psi_{x}[f, s]=\tilde{\gamma}\left(-\frac{1}{4} x[a, b]\right)(s)$. But $\psi_{x}(f)=\rho(x) f \rho(x)^{-1}$, so that

$$
\left[\psi_{x}(f), \psi_{x}(s)\right]=\tilde{\gamma} \iota\left(\rho(x) f \rho(x)^{-1}\right) \tilde{\gamma}(x)(s)=\tilde{\gamma}\left(-\frac{1}{4}[\rho(x) a, \rho(x) b] x\right)(s) .
$$

Taking into account that $\rho(x) a x=\mu(x) a x^{-1} x=x a\left(\right.$ since $\left.\left.\mu\right|_{\operatorname{Spin}(V, q)}=\mathrm{id}\right)$, we get

$$
\psi_{x}([f, s])=\left[\psi_{x}(f), \psi_{x}(s)\right] .
$$

On the other hand, $\tilde{\gamma}(x)^{-1}[f, \tilde{\gamma}(x)(s)]=\tilde{\gamma}(x)^{-1} \tilde{\gamma} \iota(f) \tilde{\gamma}(x)(s)=\tilde{\gamma}\left(\frac{-1}{4} x^{-1}[a, b] x\right)(s)=$ $\left[\rho(x)^{-1} f \rho(x), s\right]$, so that, as $(\cdot \mid \cdot)$ is $\operatorname{Spin}(V, q)$-invariant,

$$
\begin{aligned}
\operatorname{tr}\left(\left[\psi_{x}(s), \psi_{x}\left(s^{\prime}\right)\right] f\right) & =\left(\left[f, \psi_{x}(s)\right] \mid \psi_{x}\left(s^{\prime}\right)\right)=\left(\tilde{\gamma}(x)^{-1}[f, \tilde{\gamma}(x)(s)] \mid s^{\prime}\right) \\
& =\left(\left[\rho(x)^{-1} f \rho(x), s\right] \mid s^{\prime}\right)=\operatorname{tr}\left(\left[s, s^{\prime}\right] \rho(x)^{-1} f \rho(x)\right) \\
& =\operatorname{tr}\left(\rho(x)\left[s, s^{\prime}\right] \rho(x)^{-1} f\right)=\operatorname{tr}\left(\psi_{x}\left(\left[s, s^{\prime}\right]\right) f\right)
\end{aligned}
$$

and, as $f$ is arbitrary, consequently $\left[\psi_{x}(s), \psi_{x}\left(s^{\prime}\right)\right]=\psi_{x}\left(\left[s, s^{\prime}\right]\right)$. We have proved, then, that $\psi_{x} \in \operatorname{aut}\left(\mathfrak{f}_{4}\right)$.

Now note that if $F \in \operatorname{Cent}_{\text {aut }\left(\mathfrak{f}_{4}\right)}(\varphi)$ such that $\left.F\right|_{\mathfrak{b}_{4}}=\operatorname{id}_{\mathfrak{b}_{4}}$, then $F \in\left\{\operatorname{id}_{\mathfrak{f}_{4}}, \varphi\right\}$. Indeed, $\left.F\right|_{S} \in \operatorname{hom}_{\mathfrak{b}_{4}}(S, S)=\mathbb{K}$ id $_{S}$ by Schur's Lemma, so there is $\beta \in \mathbb{K}$ such that $\left.F\right|_{S}=\beta \operatorname{id}_{S}$, but, as $[S, S]=\mathfrak{b}_{4}$, that scalar $\beta \in\{1,-1\}$ and so $F$ is respectively $\{\mathrm{id}, \varphi\}$. Let us see the epimorphic character of $\psi$ : if $F \in \operatorname{Cent}_{\text {aut }\left(\mathfrak{f}_{4}\right)}(\varphi)$, we can find $x \in \operatorname{Spin}(V, q)$ such that $\psi_{x}=F$. Indeed, as $F$ commutes with $\varphi$, it preserves the $\mathbb{Z}_{2}$-grading, so we can consider the restriction $\left.F\right|_{\mathfrak{b}_{4}} \in \operatorname{aut}\left(\mathfrak{b}_{4}\right)=\operatorname{Ad}(\operatorname{SO}(V, q))$. Hence there is $A \in \mathrm{SO}(V, q)$ such that $\operatorname{Ad} A=\left.F\right|_{\mathfrak{b}_{4}}$. Take $x \in \rho^{-1}(A)$, so that $\rho^{-1}(A)=\{ \pm x\}$. Thus $\left.F^{-1} \circ \psi_{x}\right|_{\mathfrak{b}_{4}}=\mathrm{id}_{\mathfrak{b}_{4}}$ and, as above, $F^{-1} \circ \psi_{x} \in\left\{\mathrm{id}=\psi_{1}\right.$, $\left.\varphi=\psi_{-1}\right\}$. Hence $F \in\left\{\psi_{x}, \psi_{-x}\right\}$.

Finally let us check that $\psi$ is injective. If $\psi_{x}=\mathrm{id}_{\mathfrak{f}_{4}}$, then $\operatorname{Ad} \rho(x)=\mathrm{id}_{\mathfrak{b}_{4}}$, and $\rho(x) f=f \rho(x)$ for all $f \in \operatorname{so}(V, q)$. Thus $\rho(x)=\operatorname{id}_{V}$ and $x \in \operatorname{ker}(\rho)=\{ \pm 1\}$. The possibility $x=-1$ does not occur since $\psi_{-1}=\varphi \neq \mathrm{id}_{\mathfrak{f}_{4}}$. 


\subsection{Every 2-group lives in $\operatorname{Spin}(V, q)$}

We would like to prove that every MAD-group with a nontoral 2-group contains some automorphism conjugated to the automorphism $\varphi$ described in Equation (4.4), that is, some automorphism whose fixed subalgebra is of type $\mathfrak{b}_{4}$. I acknowledge A. Viruel for the communication of this result. For its proof, first recall a well known fact.

Lemma 4.2 (Lemma 3.1 in [21]). Fix a maximal torus $T \subset \operatorname{aut}(\mathcal{L})$ for $\mathcal{L}$ a semisimple Lie algebra, and an element $f \in T$. Let $W=\mathrm{N}_{\text {aut }(\mathcal{L})}(T) / T$ and $W_{f}=\mathrm{N}_{\text {Cent }(f)}(T) / T$ be the Weyl groups of aut $(\mathcal{L})$ and of the centralizer Cent $(f)$, respectively. Then the number of elements in $T$ conjugate (in aut $(\mathcal{L})$ ) to $f$ is just the Weyl group index $\left[W: W_{f}\right]$.

Proof. Recall that two elements in $T$ are conjugate in $\operatorname{aut}(\mathcal{L})$ if and only if they are conjugate in $\mathrm{N}_{\text {aut }(\mathcal{L})}(T)$. Thus the set of elements in $T$ conjugate to $f \in T$ is just $\left\{\sigma f \sigma^{-1} \mid \sigma \in \mathrm{N}_{\text {aut }}(\mathcal{L})(T)\right\}$, which is in bijective correspondence with the set of left classes $\left\{w W_{f} \mid w \in W\right\}$. Such bijection is given by $\sigma f \sigma^{-1} \mapsto(\sigma T) W_{f}$. Note that if two elements $\sigma, \sigma^{\prime} \in \mathrm{N}_{\text {aut }(\mathcal{L})}(T)$ verify $(\sigma T) W_{f}=\left(\sigma^{\prime} T\right) W_{f}$, there is $c \in \mathrm{N}_{\operatorname{Cent}(f)}(T)$ such that $\sigma^{\prime-1} \sigma T=c T$, hence $\sigma^{\prime-1} \sigma \subset \operatorname{Cent}(f) T \subset \operatorname{Cent}(f)$ so that $\sigma f \sigma^{-1}=\sigma^{\prime} f \sigma^{\prime-1}$.

Thus, if $W_{\text {aut }\left(\mathfrak{f}_{4}\right)}=\mathrm{N}_{\text {aut }\left(\mathfrak{f}_{4}\right)}(\mathcal{T}) / \mathcal{T}$ and $W_{\operatorname{Spin}(V, q)}=\mathrm{N}_{\operatorname{Spin}(V, q)}(\mathcal{T}) / \mathcal{T}$, then the index $\left[W_{\text {aut }\left(\mathfrak{f}_{4}\right)}: W_{\operatorname{Spin}(V, q)}\right]$ is computed easily by counting in any maximal torus of $\operatorname{aut}\left(\mathfrak{f}_{4}\right)$ how many elements are fixing a subalgebra of type $\mathfrak{b}_{4}$. Recall from [22] that there are two conjugacy classes of order 2 automorphisms in aut $\left(\mathfrak{f}_{4}\right)$, characterized by fixing subalgebras of type $\mathfrak{b}_{4}$ and $\mathfrak{c}_{3} \oplus \mathfrak{a}_{1}$, whose dimensions are 36 and 24 respectively. If $\mathfrak{h}$ is a Cartan subalgebra, $\mathfrak{f}_{4}=\mathfrak{h} \oplus\left(\oplus_{\alpha \in \Phi} L_{\alpha}\right)$ denotes the decomposition in root spaces relative to $\mathfrak{h}$ and $\Delta=\left\{\alpha_{i}\right\}_{i=1}^{4}$ is a set of simple roots of $\Phi$, a maximal torus can be described as $\left\{t_{x, y, z, u} \mid x, y, z, u \in \mathbb{K}^{\times}\right\}$, where $t=t_{x, y, z, u}$ is the automorphism determined by $\left.t\right|_{\mathfrak{h}}=\mathrm{id},\left.t\right|_{L_{\alpha_{1}}}=x \mathrm{id},\left.t\right|_{L_{\alpha_{2}}}=y \mathrm{id}$, $\left.t\right|_{L_{\alpha_{3}}}=z$ id and $\left.t\right|_{L_{\alpha_{4}}}=u$ id. As the eigenvalues are

$$
\begin{gathered}
(1,1,1,1) \cup\left(u, z, y, x, z u, y z, x y, x y z, y z u, y z^{2}, x y z u, y z^{2} u, x y z^{2}, x y z^{2} u, y z^{2} u^{2}\right. \\
\left.x y^{2} z^{2}, x y^{2} z^{2} u, x y z^{2} u^{2}, x y^{2} z^{3} u, x y^{2} z^{2} u^{2}, x y^{2} z^{3} u^{2}, x y^{2} z^{4} u^{2}, x y^{3} z^{4} u^{2}, x^{2} y^{3} z^{4} u^{2}\right)^{ \pm 1}
\end{gathered}
$$

the only choices of $(x, y, z, u) \in\{ \pm 1\}^{4}$ providing a list with $361^{\prime} s$ and $16-1$ 's are $(1,1,1,-1),(1,1,-1,1)$ and $(1,1,-1,-1)$. Hence, according to Lemma 4.2 , the index of the Weyl group of $\operatorname{Spin}(V, q)$ in the Weyl group of aut $\left(\mathfrak{f}_{4}\right)$ is 3 (of course this is known in the literature, see, for instance, page 248 of [20]). A consequence is the following.

Proposition 4.3. If a quasitorus $Q$ of aut $\left(\mathfrak{f}_{4}\right)$ is the direct product of a torus $T$ and a 2-group, then $Q$ is conjugated to a subquasitorus of $\operatorname{Spin}(V, q)$.

Proof. By Lemma 2.3, we can change $Q$ by one of its conjugated quasitori such that $T \subset \mathcal{T}$ and $Q \subset \mathrm{N}_{\text {aut }\left(\mathfrak{f}_{4}\right)}(\mathcal{T})$, where $\mathcal{T}$ is the maximal torus of $\operatorname{Spin}(V, q)$ defined in Equation (4.1), which is also a maximal torus of aut $\left(\mathfrak{f}_{4}\right)$ through the 
map $\psi$ defined in Proposition 4.1. Denote by $p: W_{\text {aut }\left(\mathfrak{f}_{4}\right)} \rightarrow W_{\text {aut }\left(\mathfrak{f}_{4}\right)} / W_{\operatorname{Spin}(V, q)}$ the projection onto the set of left classes. Let $f$ be an element in $Q$. We can take $f=f_{0} t$ with $t \in \mathcal{T}$ and $f_{0} \in \mathrm{N}_{\text {aut }\left(\mathfrak{f}_{4}\right)}(\mathcal{T})$ of order a power of 2 . Thus $f_{0} \mathcal{T} \in$ $W_{\text {aut }\left(\mathfrak{f}_{4}\right)}$ and its projection $p\left(f_{0} \mathcal{T}\right) \in W_{\text {aut }\left(\mathfrak{f}_{4}\right)} / W_{\operatorname{Spin}(V, q)}$ has order a power of 2 . But $\left[W_{\text {aut }\left(\mathfrak{f}_{4}\right)}: W_{\operatorname{Spin}(V, q)}\right]=3$, so that $p\left(f_{0} \mathcal{T}\right)=1$ and there is $f_{1} \in \mathrm{N}_{\operatorname{Spin}(V, q)}(\mathcal{T})$ such that $f_{0} \mathcal{T}=f_{1} \mathcal{T}$. Hence $f \in f_{0} \mathcal{T}=f_{1} \mathcal{T} \subset \operatorname{Spin}(V, q)$.

In other words, such $Q \leq \operatorname{aut}\left(\mathfrak{f}_{4}\right)$ commutes with an automorphism conjugated to $\varphi$ which fixes a subalgebra of type $\mathfrak{b}_{4}$, and hence it is contained in a MAD-group of $\operatorname{Spin}(V, q)$. We will compute these MAD-groups by taking advantage of the knowledge of the MAD-groups of $\mathrm{SO}(V, q)$, since the map $\rho: \operatorname{Spin}(V, q) \rightarrow \operatorname{SO}(V, q)$ will allow us to use that information.

\subsection{MAD-groups of $\mathrm{SO}(9)$}

According to [3], every grading on the Lie algebra $\operatorname{so}(V, q) \cong \mathfrak{b}_{4}$ is elementary, which means induced by the natural module $V$. Let us explain a little bit more about this concept. If we choose an arbitrary (finitely generated and abelian) group $G$, and take a decomposition $V=\oplus_{g \in G} V_{g}$ as a sum of vector subspaces (possibly some of them zero), we have a $G$-grading induced on $\operatorname{gl}(V)=\mathcal{L}=\oplus_{g \in G} \mathcal{L}_{g}$ given by $\mathcal{L}_{g}=\left\{f \in \operatorname{gl}(V) \mid f\left(V_{h}\right) \subset V_{g+h} \forall h \in G\right\}$ (although $G$ is not necessarily generated by the support). Such grading induces a $G$-grading on $\operatorname{so}(V, q)=\mathfrak{g}$ provided $\mathfrak{g}=\oplus_{g \in G}\left(\mathfrak{g} \cap \mathcal{L}_{g}\right)$. We will describe this kind of gradings simply by assigning a degree in $G$ to each element in some convenient basis of $V$.

Following the arguments in [3] or [17], it is easy to conclude that there are five fine gradings on $\operatorname{so}(V, q)$, over the universal grading groups (see [9] for the definition and details) $\mathbb{Z}^{4}, \mathbb{Z}^{3} \times \mathbb{Z}_{2}^{2}, \mathbb{Z}^{2} \times \mathbb{Z}_{2}^{4}, \mathbb{Z} \times \mathbb{Z}_{2}^{6}$ and $\mathbb{Z}_{2}^{8}$, induced by the following choices of basis and assignments of degree on the vector space $V$ :

- The $\mathbb{Z}^{4}$-grading induced by

$$
\begin{array}{llll}
e_{0} \mapsto(0000) & & & \\
u_{1} \mapsto(1000) & u_{2} \mapsto(0100) & u_{3} \mapsto(0010) & u_{4} \mapsto(0001) \\
v_{1} \mapsto(-1000) & v_{2} \mapsto(0-100) & v_{3} \mapsto(00-10) & v_{4} \mapsto(000-1)
\end{array}
$$

- The $\mathbb{Z}^{3} \times \mathbb{Z}_{2}^{2}$-grading induced by

$$
\begin{array}{lll}
e_{0} \mapsto(000 \overline{1} \overline{1}) & e_{1} \mapsto(000 \overline{1} \overline{0}) & e_{2} \mapsto(000 \overline{0} \overline{1}) \\
u_{2} \mapsto(100 \overline{0} \overline{0}) & u_{3} \mapsto(010 \overline{0} \overline{0}) & u_{4} \mapsto(001 \overline{0} \overline{0}) \\
v_{2} \mapsto(-100 \overline{0} \overline{0}) & v_{3} \mapsto(0-10 \overline{0} \overline{0}) & v_{4} \mapsto(00-1 \overline{0} \overline{0})
\end{array}
$$

- The $\mathbb{Z}^{2} \times \mathbb{Z}_{2}^{4}$-grading induced by

$$
\begin{array}{llll}
e_{0} \mapsto(00 \overline{1} \overline{1} \overline{1}) & & & \\
e_{1} \mapsto(00 \overline{1} \overline{0} \overline{0} \overline{0}) & e_{2} \mapsto(00 \overline{0} \overline{1} \overline{0} \overline{0}) & e_{3} \mapsto(00 \overline{0} \overline{0} \overline{0} \overline{0}) & e_{4} \mapsto(00 \overline{0} \overline{0} \overline{0} \overline{1}) \\
u_{3} \mapsto(10 \overline{0} \overline{0} \overline{0} \overline{0}) & v_{3} \mapsto(-10 \overline{0} \overline{0} \overline{0} \overline{0}) & u_{4} \mapsto(01 \overline{0} \overline{0} \overline{0} \overline{0}) & v_{4} \mapsto(0-1 \overline{0} \overline{0} \overline{0})
\end{array}
$$


- The $\mathbb{Z} \times \mathbb{Z}_{2}^{6}$-grading induced by

$$
\begin{array}{lll}
e_{0} \mapsto(0 \overline{1} \overline{1} \overline{1} \overline{1} \overline{1}) & e_{1} \mapsto(0 \overline{1} \overline{0} \overline{0} \overline{0} \overline{0} \overline{0}) & e_{2} \mapsto(0 \overline{0} \overline{1} \overline{0} \overline{0} \overline{0} \overline{0}) \\
e_{3} \mapsto(0 \overline{0} \overline{0} \overline{1} \overline{0} \overline{0}) & e_{4} \mapsto(0 \overline{0} \overline{0} \overline{0} \overline{0} \overline{0}) & e_{5} \mapsto(0 \overline{0} \overline{0} \overline{0} \overline{1} \overline{0}) \\
e_{6} \mapsto(0 \overline{0} \overline{0} \overline{0} \overline{0} \overline{1}) & u_{4} \mapsto(1 \overline{0} \overline{0} \overline{0} \overline{0} \overline{0} \overline{0}) & v_{4} \mapsto(-1 \overline{0} \overline{0} \overline{0} \overline{0} \overline{0})
\end{array}
$$

- The $\mathbb{Z}_{2}^{8}$-grading induced by

$$
\begin{array}{lll}
e_{0} \mapsto(\overline{1} \overline{1} \overline{1} \overline{1} \overline{1} \overline{1} \overline{1}) & e_{1} \mapsto(\overline{1} \overline{0} \overline{0} \overline{0} \overline{0} \overline{0} \overline{0} \overline{0}) & e_{2} \mapsto(\overline{0} \overline{1} \overline{0} \overline{0} \overline{0} \overline{0} \overline{0} \overline{0}) \\
e_{3} \mapsto(\overline{0} \overline{0} \overline{1} \overline{0} \overline{0} \overline{0} \overline{0} \overline{0}) & e_{4} \mapsto(\overline{0} \overline{0} \overline{0} \overline{1} \overline{0} \overline{0} \overline{0}) & e_{5} \mapsto(\overline{0} \overline{0} \overline{0} \overline{0} \overline{1} \overline{0} \overline{0} \overline{0}) \\
e_{6} \mapsto(\overline{0} \overline{0} \overline{0} \overline{0} \overline{0} \overline{1} \overline{0} \overline{0}) & e_{7} \mapsto(\overline{0} \overline{0} \overline{0} \overline{0} \overline{0} \overline{0} \overline{1} \overline{0}) & e_{8} \mapsto(\overline{0} \overline{0} \overline{0} \overline{0} \overline{0} \overline{0} \overline{0} \overline{1})
\end{array}
$$

The induced gradings on $\operatorname{so}(V, q)$ coincide with the gradings produced as the simultaneous diagonalizations relative to the following MAD-groups of $\operatorname{SO}(V, q)$ (respectively), where we are identifying the elements in $\mathrm{SO}(V, q)$ with their matrices relative to the base $B^{\prime}$ (notations as in Subsection 4.2):

- $Q_{1}=\left\{\left(\begin{array}{ccccc}1 & 0 & 0 & 0 & 0 \\ 0 & S_{\alpha} & 0 & 0 & 0 \\ 0 & 0 & S_{\beta} & 0 & 0 \\ 0 & 0 & 0 & S_{\delta} & 0 \\ 0 & 0 & 0 & 0 & S_{\epsilon}\end{array}\right) \mid \alpha, \beta, \delta, \epsilon \in \mathbb{K}^{\times}\right\}$,

- $Q_{2}=\left\langle\left\{\left(\begin{array}{ccc}-1 & 0 & 0 \\ 0 & -1 & 0 \\ 0 & 0 & I_{7}\end{array}\right),\left(\begin{array}{cccc}-1 & 0 & 0 & 0 \\ 0 & 1 & 0 & 0 \\ 0 & 0 & -1 & 0 \\ 0 & 0 & 0 & I_{6}\end{array}\right),\left(\begin{array}{cccc}I_{3} & 0 & 0 & 0 \\ 0 & S_{\beta} & 0 & 0 \\ 0 & 0 & S_{\delta} & 0 \\ 0 & 0 & 0 & S_{\epsilon}\end{array}\right) \mid \beta, \delta, \epsilon \in \mathbb{K}^{\times}\right\}\right\rangle$,

- $Q_{3}=\left\langle\left\{d_{0} d_{1}, d_{0} d_{2}, d_{0} d_{3}, d_{0} d_{4},\left(\begin{array}{ccc}I_{5} & 0 & 0 \\ 0 & S_{\delta} & 0 \\ 0 & 0 & S_{\epsilon}\end{array}\right) \mid \delta, \epsilon \in \mathbb{K}^{\times}\right\}\right\rangle$,

- $Q_{4}=\left\langle\left\{d_{0} d_{1}, d_{0} d_{2}, d_{0} d_{3}, d_{0} d_{4}, d_{0} d_{5}, d_{0} d_{6},\left(\begin{array}{cc}I_{7} & 0 \\ 0 & S_{\epsilon}\end{array}\right) \mid \epsilon \in \mathbb{K}^{\times}\right\}\right\rangle$,

- $Q_{5}=\left\langle\left\{d_{0} d_{1}, d_{0} d_{2}, d_{0} d_{3}, d_{0} d_{4}, d_{0} d_{5}, d_{0} d_{6}, d_{0} d_{7}, d_{0} d_{8}\right\}\right\rangle$.

\subsection{MAD-groups of $\operatorname{Spin}(9)$}

If $Q$ is a MAD-group of $\operatorname{Spin}(V, q)$, that is, a maximal abelian subgroup of semisimple elements, its image $\rho(Q)$ is also abelian and formed by semisimple elements, so that it lives in a MAD-group of $\mathrm{SO}(V, q)$ and there are $f \in \mathrm{SO}(V, q)$ and $i \in$ $\{1, \ldots, 5\}$ such that $\rho(Q) \subset f Q_{i} f^{-1}$. By replacing $Q$ with $g^{-1} Q g$ for $g \in \rho^{-1}(f)$, we can assume without loss of generality that such $Q \subset \rho^{-1}\left(Q_{i}\right)$. But it is easy to have concrete descriptions of generators of the group $\rho^{-1}\left(Q_{i}\right)$, taking into account that $\rho\left(e_{0} e_{i}\right)=d_{0} d_{i}$, according to Equation (4.2):

- $\rho^{-1}\left(Q_{1}\right)=\left\{s_{\alpha \beta \delta \epsilon} \mid \alpha, \beta, \delta, \epsilon \in \mathbb{K}^{\times}\right\}=\mathcal{T}$,

- $\rho^{-1}\left(Q_{2}\right)=\left\langle\left\{ \pm e_{0} e_{1}, \pm e_{0} e_{2}, s_{1 \beta \delta \epsilon} \mid \beta, \delta, \epsilon \in \mathbb{K}^{\times}\right\}\right\rangle$,

- $\rho^{-1}\left(Q_{3}\right)=\left\langle\left\{ \pm e_{0} e_{1}, \pm e_{0} e_{2}, \pm e_{0} e_{3}, \pm e_{0} e_{4}, s_{11 \delta \epsilon} \mid \delta, \epsilon \in \mathbb{K}^{\times}\right\}\right\rangle$,

- $\rho^{-1}\left(Q_{4}\right)=\left\langle\left\{ \pm e_{0} e_{1}, \pm e_{0} e_{2}, \pm e_{0} e_{3}, \pm e_{0} e_{4}, \pm e_{0} e_{5}, \pm e_{0} e_{6}, s_{111 \epsilon} \mid \epsilon \in \mathbb{K}^{\times}\right\}\right\rangle$,

- $\rho^{-1}\left(Q_{5}\right)=\left\langle\left\{ \pm e_{0} e_{1}, \pm e_{0} e_{2}, \pm e_{0} e_{3}, \pm e_{0} e_{4}, \pm e_{0} e_{5}, \pm e_{0} e_{6}, \pm e_{0} e_{7}, \pm e_{0} e_{8}\right\}\right\rangle$. 
Note that these groups $\rho^{-1}\left(Q_{i}\right) \leq \operatorname{Spin}(V, q)$ are not abelian if $i \neq 1$, whereas $\rho^{-1}\left(Q_{1}\right)$ is a 4 -dimensional maximal torus of $\operatorname{Spin}(V, q)$. The following considerations about some of their elements will be useful for us:

(i) The element $e_{i} e_{j}$ has order 4 if $i \neq j\left(\left(e_{i} e_{j}\right)^{2}=-1\right)$, and $e_{i} e_{j} e_{k} e_{l}$ has order 2 if $i, j, k, l$ are distinct.

(ii) If $i, j, k$ are distinct indices, $e_{i} e_{j}$ anticommutes with $e_{i} e_{k}$. More generally, $\left(e_{i_{1}} \ldots e_{i_{s}}\right)\left(e_{j_{1}} \ldots e_{j_{r}}\right)=(-1)^{m}\left(e_{j_{1}} \ldots e_{j_{r}}\right)\left(e_{i_{1}} \ldots e_{i_{s}}\right)$ if $m$ is the cardinal of the set $\left\{i_{1}, \ldots, i_{s}\right\} \cap\left\{j_{1}, \ldots, j_{r}\right\}$.

(iii) If some $s_{\alpha \beta \delta \epsilon}$ is in certain $\rho^{-1}\left(Q_{i}\right)$, then it belongs to the center of such $\rho^{-1}\left(Q_{i}\right)$. In particular $-1=s_{-1111}=s_{1-111}=s_{11-11}=s_{111-1}$ belongs to the center of $\rho^{-1}\left(Q_{i}\right)$ for all $i$.

Lemma 4.4. If $\sigma$ is a permutation of $J=\{0,1, \ldots, 8\}$, there is $x \in \operatorname{Spin}(V, q)$ such that $x e_{j} x^{-1} \in\left\{ \pm e_{\sigma(j)}\right\}$ for all $j \in J$.

Proof. It is enough to check the result for one transposition. For $\sigma=(1,2)$, note that

$$
\begin{aligned}
& s_{\xi 111} e_{1} s_{\xi 111}^{-1}=e_{2}, \\
& s_{\xi 111} e_{2} s_{\xi 111}^{-1}=-e_{1}, \\
& s_{\xi 111} e_{j} s_{\xi 111}^{-1}=e_{j}
\end{aligned}
$$

for any $j \in J \backslash\{1,2\}$, where $\xi$ is a square root of $\mathfrak{i}\left(\xi^{8}=1\right)$. Observe also that $s_{\xi 111}=\frac{\left(\xi+\xi^{7}\right)+\left(\xi^{3}+\xi^{5}\right) e_{1} e_{2}}{2}$, so that the element $\frac{\left(\xi+\xi^{7}\right)+\left(\xi^{3}+\xi^{5}\right) e_{i} e_{j}}{2} \in \operatorname{Spin}(V, q)$ works for interchanging an arbitrary pair of indices $\{i, j\} \subset J$.

Thus,

Theorem 4.5. If $Q$ is a MAD-group of $\operatorname{Spin}(V, q)$, then it is conjugated to one of the following quasitori:

(a) $P_{1}=\mathcal{T}$,

(b) $P_{2}=\left\langle\left\{e_{1} e_{2} e_{3} e_{4}, e_{1} e_{2} e_{5} e_{6}, e_{0} e_{1} e_{3} e_{5}, s_{111 \epsilon} \mid \epsilon \in \mathbb{K}^{\times}\right\}\right\rangle \cong \mathbb{Z}_{2}^{3} \times \mathbb{K}^{\times}$,

(c) $P_{3}=\left\langle\left\{-1, e_{1} e_{2} e_{3} e_{4}, e_{1} e_{2} e_{5} e_{6}, e_{1} e_{2} e_{7} e_{8}, e_{1} e_{3} e_{5} e_{7}\right\}\right\rangle \cong \mathbb{Z}_{2}^{5}$.

Remark 4.6. Note that these $P_{i}$ 's are actually MAD-groups of $\operatorname{Spin}(V, q)$. To be sure we have only to check that $P_{2}$ is not subconjugated to $P_{1}=\mathcal{T}$, that is, that $P_{2}^{\prime}=\left\langle\left\{e_{1} e_{2} e_{3} e_{4}, e_{1} e_{2} e_{5} e_{6}, e_{0} e_{1} e_{3} e_{5}\right\}\right\rangle$ is a nontoral group isomorphic to $\mathbb{Z}_{2}^{3}$. This is equivalent to proving that $\rho\left(P_{2}^{\prime}\right)$ is a nontoral group of $\mathrm{SO}(V, q)$. Identifying the elements in $\operatorname{so}(V, q)$ and $\mathrm{SO}(V, q)$ with their matrices relative to $B^{\prime}$, a straightforward computation shows that the set of skewsymmetric matrices of size 9 which commute with $\left\langle\left\{d_{1} d_{2} d_{3} d_{4}, d_{1} d_{2} d_{5} d_{6}, d_{0} d_{1} d_{3} d_{5}\right\}\right\rangle$ is the 1-dimensional space $\left\{\left(a_{i j}\right)_{i, j=0 \ldots 8} \mid a_{78}=-a_{87}, a_{i j}=0\right.$ otherwise $\}$. Thus the fixed component by the diagonalization produced by $\rho\left(P_{2}^{\prime}\right)$ has dimension strictly less than 4 (precisely 1 ), so that it does not contain a Cartan subalgebra and the grading is nontoral (see page 94 of [9]). 
Proof of Theorem 4.5. We can suppose that $Q$ is an abelian subgroup of some $\rho^{-1}\left(Q_{i}\right)$. Note also that $-1 \in Q$ by maximality of $Q$, since $\langle-1, Q\rangle$ is always abelian and diagonalizable.

If $i=1$, then $Q=\rho^{-1}\left(Q_{1}\right)=\mathcal{T}$ by maximality $(\mathcal{T}$ is already abelian).

If $i=2$, then $\left\{s_{1 \beta \delta \epsilon} \mid \beta, \delta, \epsilon \in \mathbb{K}^{\times}\right\} \subsetneq Q \subset\left\{s_{1 \beta \delta \epsilon}\right\} \cdot\left\{e_{0} e_{1}, e_{0} e_{2}, e_{1} e_{2}, 1\right\}$. Necessarily there is an element $x \in\left\{e_{0} e_{1}, e_{0} e_{2}, e_{1} e_{2}\right\}$ belonging to $Q$. But no other element in that set commutes with $x$, hence $Q=\left\{s_{1 \beta \delta \epsilon}\right\} \cdot\{1, x\}$. We can assume that $x=e_{1} e_{2}=s_{-\mathfrak{i} 111}$, because of the previous lemma. But then $Q \subsetneq \mathcal{T}$, which contradicts the maximality of $Q$.

If $i=3$, then $\left\{s_{11 \delta \epsilon} \mid \delta, \epsilon \in \mathbb{K}^{\times}\right\} \subsetneq Q \subset\left\{s_{11 \delta \epsilon}\right\} \cdot\left\langle\left\{e_{i} e_{j} \mid i, j=0,1, \ldots, 4\right\}\right\rangle$. There is $\bar{x}=\left(x_{1}, \ldots, x_{r}\right)$ with $x_{i}=a_{i, 1} \ldots a_{i, n_{i}}, a_{i, j} \in\left\{e_{0}, \ldots, e_{4}\right\}, n_{i}$ even, such that $Q=\left\{s_{11 \delta \epsilon}\right\} \cdot\left\langle\left\{x_{1}, \ldots, x_{r}\right\}\right\rangle$ and each $x_{j} \notin\left\{s_{11 \delta \epsilon}\right\} \cdot\left\langle\left\{x_{1}, \ldots, x_{j-1}\right\}\right\rangle$. Among the possible $\bar{x}$ verifying such conditions, choose one such that the attached $\bar{n}=\left(n_{1}, \ldots, n_{r}\right) \in \mathbb{N}^{r}$ is minimum in $\cup_{s \in \mathbb{N}} \mathbb{N}^{s}$ with the lexicographical order. In particular $n_{1} \leq \cdots \leq n_{r}$, taking into account that for any permutation $\sigma \in S_{r}, \bar{x}^{\sigma}=\left(x_{\sigma(1)}, \ldots, x_{\sigma(r)}\right)$ verifies the same conditions as $\bar{x}$. Indeed, assume that $x_{\sigma(j)} \in\left\{s_{11 \delta \epsilon}\right\} \cdot\left\langle\left\{x_{\sigma(1)}, \ldots, x_{\sigma(j-1)}\right\}\right\rangle$. Thus $x_{\sigma(j)}=s_{11 d e} x_{\sigma(1)}^{s_{1}} \ldots x_{\sigma(j-1)}^{s_{j-1}}$ for some $d, e \in \mathbb{K}^{\times}$and $s_{i} \in\{0,1\}$, since $x_{i}^{2}=(-1)^{\frac{n_{i}}{2}} \in\{ \pm 1\}$. Now we choose $k \in\{1, \ldots, j-1\}$ such that $\sigma(k)$ is the greatest index with $s_{k} \neq 0$ (necessarily $\sigma(k)>\sigma(j)$ and $\left.s_{k}=1\right)$ and then $x_{\sigma(k)}= \pm s_{11 d e} x_{\sigma(1)}^{s_{1}} \ldots \hat{x}_{\sigma(k)}^{s_{k}} \ldots x_{\sigma(j-1)}^{s_{j-1}} x_{\sigma(j)} \in$ $\left\{s_{11 \delta \epsilon}\right\} \cdot\left\langle\left\{x_{1}, \ldots, x_{\sigma(k)-1}\right\}\right\rangle$, a contradiction. As $\operatorname{Spin}(V, q)$ is a simply connected group, Lemma 2.1 and Lemma 2.2 can be applied to get that $r \geq 3$. If $n_{1}=2$, then we can assume that $x_{1}=e_{1} e_{2}$ by Lemma 4.4 , because the element used for conjugating does not change $s_{11 \delta \epsilon}$. In the same way we can assume that $x_{2}=e_{3} e_{4}$ if $n_{2}=2$ (and $x_{2}=e_{1} e_{2} e_{3} e_{4}$ if $n_{2}=4$, but then $\bar{n}$ would not be minimal). But now there is no possibility for $x_{3}$ (it should have an even -not 2- number of indices in common with $\{1,2\}$ and with $\{3,4\})$. If $n_{1}=4$, then we can assume that $x_{1}=e_{1} e_{2} e_{3} e_{4}$ but there is no $x_{2}$ with the required conditions.

If $i=4$, we have a similar situation: $\left\{s_{111 \epsilon} \mid \epsilon \in \mathbb{K}^{\times}\right\} \subsetneq Q \subset\left\{s_{111 \epsilon}\right\}$. $\left\langle\left\{e_{i} e_{j} \mid i, j=0,1, \ldots, 6\right\}\right\rangle$, so that we can take $Q=\left\{s_{111 \epsilon}\right\} \cdot\left\langle\left\{x_{1}, \ldots, x_{r}\right\}\right\rangle$ for certain $x_{j} \in \operatorname{Spin}(V, q) \backslash\left\{s_{111 \epsilon}\right\} \cdot\left\langle\left\{x_{1}, \ldots, x_{j-1}\right\}\right\rangle$ product of $n_{j} \in\{2,4,6\}$ elements in $\left\{e_{0}, \ldots, e_{6}\right\}$. Again the $r \geq 3$ generators have been chosen such that $\bar{n}=$ $\left(n_{1}, \ldots, n_{r}\right)$ is minimum, and, in particular, $n_{1} \leq \cdots \leq n_{r}$. If $n_{1}=n_{2}=2$, then we can assume that $x_{1}=e_{1} e_{2}=s_{-i 111}$ and that $x_{2}=e_{3} e_{4}$, again by Lemma 4.4 . As the $e_{i}$ 's involved in $x_{3}$ are only $e_{0}, e_{5}, e_{6}$ (otherwise there would be another $\bar{x}^{\prime}$ with $n_{3}^{\prime}<n_{3}$ so that $\bar{n}^{\prime}=\left(n_{1}, n_{2}, n_{3}^{\prime}, \ldots\right)$ is lesser than $\left.\bar{n}\right)$, this implies that $n_{3}=2$, so that we can assume that $x_{3}=e_{5} e_{6}$. But nothing more in $\rho^{-1}\left(Q_{4}\right)$ commutes with all these elements, hence $Q=\left\{s_{111 \epsilon}\right\} \cdot\left\langle\left\{s_{-\mathfrak{i} 111}, s_{1-\mathfrak{i} 11}, s_{11-\mathfrak{i} 1}\right\}\right\rangle$, which is strictly contained in $\mathcal{T}$, a contradiction. If $n_{1}=2$ and $n_{2}=4$ we can assume that $x_{1}=e_{1} e_{2}$ and that $x_{2}=e_{3} e_{4} e_{5} e_{6}$. Now there is no $x_{3}$ satisfying the conditions (with at least four $e_{i}$ 's involved, then $e_{1}$ and $e_{2}$ would appear and we could lessen $n_{3}$ in $\bar{n}$ ). Neither there is any possibility with $n_{1}=2$ and $n_{2}=6$. Hence $n_{1}=4$. That forces $n_{2}=4=n_{3}$ because if some $n_{i}=6, x_{i}$ would have four indices in common with $x_{1}$ (there are not enough elements for having only two in common) and $x_{1} x_{i}$ would have only two involved elements (getting $\bar{n}^{\prime}$ less than $\bar{n}$ 
again). So we can assume that $x_{1}=e_{1} e_{2} e_{3} e_{4}$, that $x_{2}=e_{1} e_{2} e_{5} e_{6}$, and that $x_{3}$ has just two $e_{i}$ 's in common with $x_{1}$ and 2 with $x_{2}$. These elements cannot be $e_{1}$ and $e_{2}$ (there is only $e_{0}$ to add) so that there are in $x_{3}$ one element in $\left\{e_{1}, e_{2}\right\}$, one element in $\left\{e_{3}, e_{4}\right\}$ and one element in $\left\{e_{5}, e_{6}\right\}$, and consequently we can assume that $x_{3}=e_{0} e_{1} e_{3} e_{5}$. Now $P_{2} \subset Q$, but the only elements in $\rho^{-1}\left(Q_{4}\right)$ commuting with $P_{2}$ belong to $P_{2}$, so that $P_{2}=Q$.

If $i=5$, we can take similarly to the previous cases $Q=\left\langle\left\{-1, x_{1}, \ldots, x_{r}\right\}\right\rangle$, where each $x_{j}$ is a product of an even number $n_{j} \in\{2,4,6,8\}$ of elements in $\left\{e_{0}, \ldots, e_{8}\right\}$, satisfying that $x_{j} \notin\left\langle\left\{-1, x_{1}, \ldots, x_{j-1}\right\}\right\rangle, \bar{n}=\left(n_{1}, \ldots, n_{r}\right)$ minimum, $n_{1} \leq \cdots \leq n_{r}$ and $r \geq 3$. If $\bar{n}=(2,2,2, \ldots)$, then we can change the generators by $x_{1}=e_{1} e_{2}, x_{2}=e_{3} e_{4}, x_{3}=e_{5} e_{6}$ and then necessarily $n_{4}=2$ and we can take $x_{4}=e_{7} e_{8}$. Thus nothing more can be added and $Q \subset \mathcal{T}$. If $\bar{n}=(2,2,4, \ldots)$, then we can change the generators into $x_{1}=e_{1} e_{2}, x_{2}=e_{3} e_{4}$, $x_{3}=e_{5} e_{6} e_{7} e_{8}$ and again nothing more can be added and $Q \subset \mathcal{T}$. The choice $\bar{n}=(2,2,6, \ldots)$ would not provide $\bar{n}$ minimal. If $\bar{n}=(2,4, \ldots)$, then we can change the generators into $x_{1}=e_{1} e_{2}$ and $x_{2}=e_{3} e_{4} e_{5} e_{6}$. If $n_{3}=4$, we can take $x_{3}=e_{5} e_{6} e_{7} e_{8}$, so that also $n_{4}=4$ and $x_{4}$ has two elements in $\{3,4,5,6\}$ and two in $\{5,6,7,8\}$ (none in $\{1,2\}$ ). Thus we can take $x_{4}=e_{0} e_{3} e_{5} e_{7}$ and necessarily $Q=\left\langle\left\{-1, e_{1} e_{2}, e_{3} e_{4} e_{5} e_{6}, e_{5} e_{6} e_{7} e_{8}, e_{0} e_{3} e_{5} e_{7}\right\}\right\rangle$, which is not a MADgroup, because according to Lemma 4.4 the element $x \in \operatorname{Spin}(V, q)$ related to the permutation $\sigma=\left(\begin{array}{lllllllll}0 & 1 & 2 & 3 & 4 & 5 & 6 & 7 & 8 \\ 0 & 7 & 8 & 3 & 4 & 1 & 2 & 5 & 6\end{array}\right)$ verifies that $x Q x^{-1}$ is strictly contained in $P_{2}$. Of course the case $n_{3}=6$ is not possible and we conclude that $n_{1}=4$. Now we get that $n_{1}=n_{2}=n_{3}=4$ and modify the generators to be either $\left(x_{1}, x_{2}, x_{3}\right)=\left(e_{1} e_{2} e_{3} e_{4}, e_{1} e_{2} e_{5} e_{6}, e_{1} e_{2} e_{7} e_{8}\right)$ or $\left(e_{1} e_{2} e_{3} e_{4}, e_{1} e_{2} e_{5} e_{6}, e_{1} e_{3} e_{5} e_{7}\right)$. The generated groups are different, even though both are isomorphic to $\mathbb{Z}_{2}^{3}$ as abstract groups, the first one is obviously toral (just contained in $\mathcal{T}$ ), but the second one is nontoral according to Remark 4.6 (we talked there about $e_{0} e_{1} e_{3} e_{5}$ as in case $i=4$, but we can map $e_{0} e_{1} e_{3} e_{5}$ into $\pm e_{1} e_{3} e_{5} e_{7}$ without moving $e_{1}, \ldots, e_{6}$ by Lemma 4.4). In both cases there is a fourth element in $\rho^{-1}\left(Q_{5}\right)$ commuting with them: $x_{4}=e_{1} e_{3} e_{5} e_{7}$ and $x_{4}=e_{1} e_{2} e_{7} e_{8}$ respectively, which obviously leads us to the same $Q$. Now there is no possibility of adding anything else, so that $r=4$.

Note that $\varphi=\psi_{-1} \in \psi\left(P_{i}\right)$ for all $i=1,2,3$. They are the only MAD-groups containing $\varphi$ :

Corollary 4.7. If $Q$ is a MAD-group of aut $\left(\mathfrak{f}_{4}\right)$ which contains $\varphi$, then $Q$ is conjugated to

(a) $\psi\left(P_{1}\right) \cong\left(\mathbb{K}^{\times}\right)^{4}$,

(b) $\psi\left(P_{2}\right) \cong \mathbb{Z}_{2}^{3} \times \mathbb{K}^{\times}$,

(c) $\psi\left(P_{3}\right) \cong \mathbb{Z}_{2}^{5}$.

Proof. As $\varphi \in Q, Q$ is contained in $\operatorname{Cent}_{\text {aut }\left(\mathfrak{f}_{4}\right)}(\varphi)$, which, according to Proposition 4.1 , coincides with $\psi(\operatorname{Spin}(V, q))$. Taking into account that $\psi$ is an isomorphism, Theorem 4.5 gives the result. 


\section{3-groups of $\operatorname{aut}\left(\mathfrak{f}_{4}\right)$}

The objective here is to prove

Theorem 5.1. There is an only nontoral 3-subgroup of $\operatorname{aut}\left(\mathfrak{f}_{4}\right)$. It is isomorphic to $\mathbb{Z}_{3}^{3}$ as abstract group. It is a MAD-group.

There are several results in the literature related to this one, as

Proposition 5.2 (Proposition 3.5 in [27], more detailed in Theorem (7.4) of [16]). There is an only nontoral elementary 3 -group of aut $\left(\mathfrak{f}_{4}\right)$. It is isomorphic to $\mathbb{Z}_{3}^{3}$ as an abstract group. It is a MAD-group.

The problem is that we cannot conclude Theorem 5.1 from this proposition, at least not directly, as we observed in Remark 2.10. On the other hand, the computational methods do not turn out to be difficult for this prime, but precisely our main aim in this paper is to avoid completely the usage of computer. Thus, we proceed as in the case of the prime 2, following similar steps: a nontoral 3-group must contain some order 3 automorphism fixing a subalgebra of type $\mathfrak{a}_{2} \oplus \mathfrak{a}_{2}$ and hence it lives in the corresponding centralizer, certain quotient of $\operatorname{SL}(3)^{2}$. Then we try to use our knowledge of the gradings on matrix algebras.

\subsection{Model of $\mathfrak{f}_{4}$ based on $2 \mathfrak{a}_{2}$}

Now let $V$ and $W$ be 3-dimensional vector spaces and take

$$
\mathcal{L}=\mathcal{L}_{\overline{0}} \oplus \mathcal{L}_{\overline{1}} \oplus \mathcal{L}_{\overline{2}}
$$

the $\mathbb{Z}_{3}$-graded Lie algebra given by

$$
\mathcal{L}_{\overline{0}}=\operatorname{sl}(V) \oplus \operatorname{sl}(W), \quad \mathcal{L}_{\overline{1}}=V \otimes S^{2}(W), \quad \mathcal{L}_{\overline{2}}=V^{*} \otimes S^{2}\left(W^{*}\right),
$$

where $S^{2}(U)$ denotes the symmetric tensors in $U \otimes U$ and the product is given in the following way:

- $\operatorname{sl}(V) \oplus \operatorname{sl}(W)$ is a Lie subalgebra.

- The actions of $\mathcal{L}_{\overline{0}}$ on $V \otimes S^{2}(W)$ and on $V^{*} \otimes S^{2}\left(W^{*}\right)$ are the natural ones.

- We have fixed a nonzero trilinear alternating map det: $V \times V \times V \rightarrow \mathbb{K}$ so that we identify $V \wedge V$ with $V^{*}$ by means of $u \wedge v \mapsto \operatorname{det}(u, v,-)$. For det ${ }^{*}$ the dual map of det, we also identify $V^{*} \wedge V^{*}$ with $V$. Proceed similarly with $W$. Now for any $u, v \in V, w, x \in W, f, g \in V^{*}, h, j \in W^{*}$, and denoting by $f_{u}$ the endomorphism $f(-) u \in \operatorname{gl}(V)$ and by $\pi f \equiv f-\frac{1}{3} \operatorname{tr}(f)$ id the projection on the traceless endomorphisms,

$$
\begin{aligned}
& {[f \otimes h \cdot h, u \otimes w \cdot w]=h(w)^{2} \pi f_{u}+f(u) h(w) \pi h_{w},} \\
& {[u \otimes w \cdot w, v \otimes x \cdot x]=(u \wedge v) \otimes(w \wedge x) \cdot(w \wedge x),} \\
& {[f \otimes h \cdot h, g \otimes j \cdot j]=(f \wedge g) \otimes(h \wedge j) \cdot(h \wedge j) .}
\end{aligned}
$$


The so described algebra is simple of type $\mathfrak{f}_{4}$ (see [8] for details about this and other constructions of $\mathfrak{f}_{4}$ ). Call $\phi$ the order 3 grading automorphism. We compute its centralizer. Note that now the adjoint map denotes $\mathrm{Ad}: \mathrm{SL}(V) \rightarrow \operatorname{gl}(\operatorname{sl}(V))$ given by $\operatorname{Ad} x(f)=x f x^{-1}$ for any $x \in \mathrm{SL}(V), f \in \operatorname{sl}(V) \equiv \mathfrak{a}_{2}$, and similarly for $W$. For $x \in \mathrm{SL}(V), y \in \mathrm{SL}(W)$, consider the map $\Psi_{x, y}: \mathfrak{f}_{4} \rightarrow \mathfrak{f}_{4}$ given by $\left.\Psi_{x, y}\right|_{\mathrm{sl}(V)}=\operatorname{Ad} x$, $\left.\Psi_{x, y}\right|_{\operatorname{sl}(W)}=\operatorname{Ad} y, \Psi_{x, y}\left(v \otimes w_{1} \cdot w_{2}\right)=(x \cdot v) \otimes\left(y \cdot w_{1}\right) \cdot\left(y \cdot w_{2}\right)$ for any $v \in V$ and $w_{1}, w_{2} \in W$, and $\Psi_{x, y}\left(f \otimes g_{1} \cdot g_{2}\right)=(x \cdot f) \otimes\left(y \cdot g_{1}\right) \cdot\left(y \cdot g_{2}\right)$ for any $f \in V^{*}$ and $g_{1}, g_{2} \in W^{*}$, where denotes the symmetric product as well as the action of SL on its natural and dual representations.

Proposition 5.3. The map $\Psi_{x, y}$ is an automorphism of the Lie algebra $\mathcal{L}=\mathfrak{f}_{4}$ for all $x \in \mathrm{SL}(V)$ and $y \in \mathrm{SL}(W)$; and the map

$$
\Psi: \operatorname{SL}(V) \times \mathrm{SL}(W) \rightarrow \operatorname{Cent}_{\mathrm{aut}_{\left(\mathfrak{f}_{4}\right)}(\phi)}
$$

given by $(x, y) \mapsto \Psi_{x, y}$ is a group epimorphism with kernel $\left\{\left(\omega^{n} \mathrm{id}_{V}, \omega^{n} \mathrm{id}_{W}\right)\right.$ : $n=0,1,2\} \cong \mathbb{Z}_{3}$, for $\omega$ a primitive cubic root of the unit.

Proof. Proceed as in the proof of Proposition 4.1 to check that this is a well defined surjective map, and of course a group homomorphism. Let us compute the kernel. If $\Psi_{x, y}=\mathrm{id}_{\mathfrak{f}_{4}}$, the element $x$ commutes with $\operatorname{sl}(V)$, and hence there is $\alpha \in \mathbb{K}$ such that $x=\alpha \operatorname{id}_{V}$. But, as $\operatorname{det}(x)=1$, necessarily $\alpha^{3}=1$. In the same way, $y=\beta \operatorname{id}_{W}$ with $\beta^{3}=1$. Now $\left.\Psi_{x, y}\right|_{\mathcal{L}_{\overline{1}}}=\alpha \beta^{2}$ id, so that $\alpha \beta^{2}$ must be equal to 1 and hence $\alpha=\beta$.

\subsection{Every 3-group lives in $\operatorname{SL}(3)^{2} / \mathbb{Z}_{3}$}

We would like to prove that every nontoral 3-group contains some automorphism conjugated to $\phi$.

According to [20], page 248, the index of the Weyl group of $\operatorname{Cent}_{\text {aut }\left(\mathfrak{f}_{4}\right)}(\phi)$ in the Weyl group of aut $\left(\mathfrak{f}_{4}\right)$ is 32 (this number can also be easily computed with the trick described in Lemma 4.2), coprime to 3. Again this fact implies that

Proposition 5.4. If $Q$ is a 3-group of aut $\left(\mathfrak{f}_{4}\right)$, then $Q$ is conjugated to a subquasitorus of $\operatorname{Cent}_{\text {aut }\left(\mathfrak{f}_{4}\right)}(\phi)$.

Which can be proved analogously to Proposition 4.3.

\subsection{MAD-groups of $\mathrm{SL}(3)$}

Proposition 5.5. There are four fine gradings on the algebra sl(3). Their grading groups are

$$
\mathbb{Z}^{2}, \quad \mathbb{Z} \times \mathbb{Z}_{2}, \quad \mathbb{Z}_{2}^{3}, \quad \mathbb{Z}_{3}^{2} .
$$

Equivalently, up to conjugation there are four MAD-groups of

$$
\operatorname{aut}(\operatorname{sl}(3)) \cong \operatorname{PSL}(3) \rtimes \mathbb{Z}_{2}
$$


This result can be concluded from [5], but the gradings are explicitly computed in [18]. We do not really need a concrete description of all the gradings, it is enough for our purposes to recall which is the $\mathbb{Z}_{3}^{2}$-nontoral grading. If we denote by

$$
b:=\left(\begin{array}{ccc}
1 & 0 & 0 \\
0 & \omega & 0 \\
0 & 0 & \omega^{2}
\end{array}\right), \quad c:=\left(\begin{array}{ccc}
0 & 0 & 1 \\
1 & 0 & 0 \\
0 & 1 & 0
\end{array}\right)
$$

we can observe that $b$ and $c$ are elements of order 3 in SL(3) that do not commute: $b c=\omega c b$. On the contrary, their classes in $\operatorname{PSL}(3)=\mathrm{SL}(3) /\left\langle\omega I_{3}\right\rangle$ do commute, and $\langle\{\bar{b}, \bar{c}\}\rangle \cong \mathbb{Z}_{3}^{2}$ is a MAD-group of $\operatorname{PSL}(3)$, where $\bar{x}$ denotes the class of the element $x \in \mathrm{SL}(3)$ modulo $\left\langle\omega I_{3}\right\rangle$.

Identify $\mathrm{SL}(V)$ and $\mathrm{SL}(W)$ with $\mathrm{SL}(3)$ by means of their matrices relative to some fixed bases and also identify

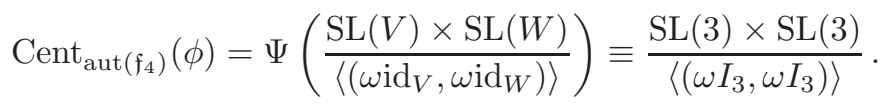

Now consider the projections

$$
\pi_{i}: \frac{\mathrm{SL}(3) \times \mathrm{SL}(3)}{\left\langle\left(\omega I_{3}, \omega I_{3}\right)\right\rangle} \rightarrow \operatorname{PSL}(3)=\frac{\mathrm{SL}(3)}{\left\langle\omega I_{3}\right\rangle}
$$

given by $\pi_{1}\left(\Psi_{[x ; y]}\right)=\bar{x}$ and $\pi_{2}\left(\Psi_{[x ; y]}\right)=\bar{y}$, where $[x ; y]$ denotes the class of the element $(x, y) \in \mathrm{SL}(3) \times \mathrm{SL}(3)$ modulo $\left\langle\left(\omega I_{3}, \omega I_{3}\right)\right\rangle$. Note that they are well defined because $\pi_{i}\left(\Psi_{\left[\omega I_{3} ; \omega I_{3}\right]}\right)=\overline{\omega I_{3}}=\bar{I}_{3}$.

Proof of Theorem 5.1. Take $Q$ a nontoral 3-group, which can be assumed contained in $\operatorname{Cent}_{\text {aut }\left(\mathfrak{f}_{4}\right)}(\phi)$, so that each $\pi_{i}(Q)$ is a subquasitorus of aut $(\mathrm{sl}(3))$ which lives in $\left(\mathbb{K}^{\times}\right)^{2},\left(\mathbb{K}^{\times}\right) \times \mathbb{Z}_{2}, \mathbb{Z}_{2}^{3}$ or $\mathbb{Z}_{3}^{2}$ by Proposition 5.5 . It is clear, as in Lemma 2.5, that $\pi_{i}(Q)$ is contained in $\left(\mathbb{K}^{\times}\right)^{2}, \mathbb{K}^{\times}$, id or $\mathbb{Z}_{3}^{2}$. But $\pi_{i}$ maps nontoral groups into nontoral groups, so that we can also assume that $\pi_{i}(Q)=\langle\{\bar{b}, \bar{c}\}\rangle \cong \mathbb{Z}_{3}^{2}$. Now, an arbitrary element in $Q$ is $\Psi_{[x ; y]}$ with $x, y \in\left\{\omega^{n_{1}} b^{n_{2}} c^{n_{3}} \mid n_{i}=0,1,2\right\}=: P$. Hence $x^{3}=y^{3}=I_{3}$, the element $\Psi_{[x ; y]}$ has order 3 and $Q$ is elementary, so that we could apply Proposition 5.2 to finish our proof. But, again for selfcontainedness, we are going to prove that

$$
Q \cong\left\langle\left\{\Psi_{\left[I_{3} ; \omega I_{3}\right]}, \Psi_{[b ; b]}, \Psi_{[c ; c]}\right\}\right\rangle=: Q^{\prime} .
$$

Take some elements $\Psi_{\left[b ; y_{1}\right]} \in \pi_{1}^{-1}(\bar{b})$ and $\Psi_{\left[c ; y_{2}\right]} \in \pi_{1}^{-1}(\bar{c})$. They commute, so that $\left[b c=\omega c b ; y_{1} y_{2}\right]=\left[c b ; y_{2} y_{1}\right]$ and $y_{1} y_{2}=\omega y_{2} y_{1}$. In particular, $y_{1} \notin\left\{I_{3}, \omega I_{3}, \omega^{2} I_{3}\right\}$. As $\Psi_{\left[I_{3} ; y\right]} \Psi_{\left[b ; y_{1}\right]} \Psi_{\left[I_{3} ; y\right]}^{-1}=\Psi_{\left[b ; y y_{1} y^{-1}\right]}$, we can replace $y_{1}$ by $b$ (the 26 order 3 elements in $P$ are conjugated in SL(3)). This implies that $y_{2}=\omega^{n_{1}} b^{n_{2}} c$. As $\left\langle\left\{\Psi_{[b ; b]}, \Psi_{\left[c ; y_{2}\right]}\right\}\right\rangle$ is toral (arguments as in Lemma 2.1), we can find $\Psi_{\left[x_{3} ; y_{3}\right]} \in Q \backslash\left\langle\left\{\Psi_{[b ; b]}, \Psi_{\left[c ; y_{2}\right]}\right\}\right\rangle$. We can assume that $x_{3}=I_{3}$ (if $x_{3}=b$, replace it by $\Psi_{\left[x_{3} ; y_{3}\right]} \Psi_{[b ; b]}^{2}$, and do the same for any of the other possibilities for $x_{3}$ ). Now, the commutativity condition forces $y_{3}$ to commute with $b$ and $b^{n_{2}} c$, hence $y_{3} \in\left\{1, \omega, \omega^{2}\right\} I_{3}$. But $y_{3} \neq I_{3}$, so 
$\Psi_{\left[I_{3} ; \omega I_{3}\right]} \in Q$. Thus $\left\langle\left\{\Psi_{\left[I_{3} ; \omega I_{3}\right]}, \Psi_{[b ; b]}, \Psi_{\left[c ; b^{n_{2}} c\right]}\right\}\right\rangle \subset Q$. Note now that the diagonal matrix $p=\operatorname{diag}\left\{1, \omega^{2}, 1\right\} \in \mathrm{SL}(3)$ verifies that $p b p^{-1}=b$ and $p c p^{-1}=b c$, so that $Q^{\prime}$ is contained in a quasitorus conjugated to $Q$ (by means of $\Psi_{\left[I_{3} ; p\right]}$ or $\Psi_{\left[I_{3} ; p^{2}\right]}$ ), but $Q^{\prime}$ is its own centralizer and we are done.

\section{MAD-groups of $\operatorname{aut}\left(\mathfrak{f}_{4}\right)$}

Lemma 6.1. The automorphisms $\psi_{ \pm e_{1} e_{2} e_{3} e_{4} e_{5} e_{6} e_{7} e_{8}}$ are conjugated to $\varphi$.

Proof. Recall that any order 2 automorphism in aut $\left(\mathfrak{f}_{4}\right)$ fixes a subalgebra of type either $\mathfrak{b}_{4}$ or $\mathfrak{c}_{3} \oplus \mathfrak{a}_{1}$, so that the conjugacy class is determined by the dimension of the fixed part of any representative in the class (36 and 24 respectively). Thus we have only to check that $\operatorname{dim} \operatorname{Fix}\left(\varphi_{i}\right)=36$ for

$$
\varphi_{1}=\psi_{e_{1} e_{2} e_{3} e_{4} e_{5} e_{6} e_{7} e_{8}} \quad \text { and } \quad \varphi_{2}=\psi_{-e_{1} e_{2} e_{3} e_{4} e_{5} e_{6} e_{7} e_{8}}=\varphi_{1} \varphi .
$$

First note that the restriction to the even part $\left.\varphi_{i}\right|_{\mathrm{so}(V, q)}=\operatorname{Ad} \rho\left(e_{1} e_{2} e_{3} e_{4} e_{5} e_{6} e_{7} e_{8}\right)$ fixes the subalgebra $\operatorname{so}\left(V^{\prime}, q\right)$ for $V^{\prime}=\operatorname{span}\left\langle\left\{e_{1}, e_{2}, e_{3}, e_{4}, e_{5}, e_{6}, e_{7}, e_{8}\right\}\right\rangle$, which is a Lie algebra of type $\mathfrak{d}_{4}$ and dimension 28 .

In order to compute the fixed part of $\left.\varphi_{i}\right|_{\wedge W}$, note that $\tilde{\gamma}\left(e_{1} e_{2}\right)=\tilde{\gamma}\left(\frac{i}{2}\left[v_{1}, u_{1}\right]\right)=$ $-2 \mathfrak{i} \tilde{\gamma} \iota\left(h_{1}\right)$ and, taking into account Equation (4.3), if $s=u_{j_{1}} \wedge \cdots \wedge u_{j_{r}}$,

$$
\varphi_{1}(s)=-\varphi_{2}(s)=(2 \mathfrak{i})^{4} h_{1} \cdot\left(h_{2} \cdot\left(h_{3} \cdot\left(h_{4} \cdot s\right)\right)\right)=(-1)^{n_{1}+n_{2}+n_{3}+n_{4}} s
$$

where $n_{i}=0$ if $i \in\left\{j_{1}, \ldots, j_{r}\right\}$ and $n_{i}=1$ otherwise. Hence $\sum_{i=1}^{4} n_{i}=4-r$ and $\varphi_{1}(s)=s$ just when $r$ is even. This means that $\operatorname{Fix} \varphi_{1}=\wedge_{\overline{0}} W$ and $\operatorname{Fix} \varphi_{2}=\wedge_{\overline{1}} W$, so that $\left.\operatorname{dim} \operatorname{Fix} \varphi_{i}\right|_{\wedge W}=8$ and $\operatorname{dim} \operatorname{Fix} \varphi_{i}=36$, as desired.

Theorem 6.2. The fine gradings on $\mathfrak{f}_{4}$ are, up to equivalence, the four fine gradings described in Section 3.

Proof. Take $Q$ a MAD-group of aut $\left(\mathfrak{f}_{4}\right)$ different from the maximal torus. If $Q$ contains a nontoral 3-group $R_{3}$, then $R_{3}$ is itself a MAD-group by Theorem 5.1, and hence $Q=R_{3}$. Otherwise $Q$ contains $R_{2}$ a nontoral 2-group by Corollary 2.11. Let us show that in this case $Q$ is conjugated to either $\psi\left(P_{2}\right)$ or $\psi\left(P_{3}\right)$, where $P_{2}$ and $P_{3}$ are described in Theorem 4.5. According to Corollary 2.13, we are in the following situation: $Q=T \times R_{2} \times R$ with $T$ a torus, $R_{2}$ a nontoral 2-group and $R$ a finite group of odd order. Now, by Proposition 4.3, we can assume that $T \times R_{2} \subset \psi(\operatorname{Spin}(V, q))$ and, by Theorem 4.5, that $T \times R_{2}$ is contained in either $\psi\left(P_{2}\right) \cong \mathbb{Z}_{2}^{3} \times \mathbb{K}^{\times}$or $\psi\left(P_{3}\right) \cong \mathbb{Z}_{2}^{5}$. If $R$ is trivial, then $Q=T \times R_{2} \subset \psi(\operatorname{Spin}(V, q))$ and we have finished by Corollary 4.7. We are also done if $\varphi=\psi(-1) \in Q$, since then $Q \subset \operatorname{Cent}_{\text {aut }\left(\mathfrak{f}_{4}\right)}(\varphi)=\psi(\operatorname{Spin}(V, q))$ (of course in this case $R$ turns out to be trivial). If $R$ is not trivial, by Corollary 2.13 the 2-group $R_{2}$ has at least 4 factors. If $R_{2} \subset \psi\left(P_{2}\right)$, there is $\epsilon \in \mathbb{K}^{\times}$of order a power of 2 (root of the unit) such that $\psi^{-1}\left(R_{2}\right)=\left\langle\left\{e_{1} e_{2} e_{3} e_{4}, e_{1} e_{2} e_{5} e_{6}, e_{0} e_{1} e_{3} e_{5}, s_{111 \epsilon}\right\}\right\rangle$. Thus we have the contradiction $-1=s_{111-1} \in \psi^{-1}\left(R_{2}\right)$. The other possibility is that $R_{2}$ is contained in $\psi\left(P_{3}\right)$. As 
$\psi(-1) \notin R_{2}$, the existence of the four factors forces $R_{2}$ to be the image under $\psi$ of $\left\langle\left\{\alpha_{1} e_{1} e_{2} e_{3} e_{4}, \alpha_{2} e_{1} e_{2} e_{5} e_{6}, \alpha_{3} e_{1} e_{2} e_{7} e_{8}, \alpha_{4} e_{1} e_{3} e_{5} e_{7}\right\}\right\rangle$ for certain scalars $\alpha_{j} \in\{ \pm 1\}$. Hence there is $\alpha \in\{ \pm 1\}$ such that $\psi\left(\alpha e_{1} e_{2} e_{3} e_{4} e_{5} e_{6} e_{7} e_{8}\right) \equiv \varphi^{\prime}$ belongs to $R_{2}$. According to the previous lemma, $\varphi^{\prime}$ is conjugated to $\varphi$ and this finishes the proof.

\section{References}

[1] Andersen, K. K.S., Grodal, J., Møller, J. M. and Viruel, A.: The classification of $p$-compact groups for $p$ odd. Ann. of Math. (2) 167 (2008), no. 1, 95-210.

[2] Bahturin, Y.A. And Kotchetov, M.: Classification of group gradings on simple Lie algebras of types $A, B, C$ and D. J. Algebra 324 (2010), no. 11, 2971-2989.

[3] Bahturin, Y.A., Shestakov, I. P. and Zaicev, M. V.: Gradings on simple Jordan and Lie algebras. J. Algebra 283 (2005), no. 2, 849-868.

[4] Bahturin, Y.A. And Tvalavadze, M.: Group gradings on $G_{2}$. Comm. Algebra 37 (2009), no. 3, 885-893.

[5] Bahturin, Y.A. And Zaicev, M. V.: Group gradings on simple Lie algebras of type "A". J. Lie Theory 16 (2006), no. 4, 719-742.

[6] Borel, A.: Linear algebraic groups. Second enlarged edition. Graduate Texts in Mathematics 126, Springer-Verlag, New York, 1991.

[7] Carter, R. W.: Finite groups of Lie type. Conjugacy classes and complex characters. Pure and Applied Mathematics, John Wiley, New York, 1985.

[8] Draper, C.: Models of the Lie algebra $F_{4}$. Linear Algebra Appl. 428 (2008), no. 11-12, 2813-2839.

[9] Draper, C. and Martín, C.: Gradings on $\mathfrak{g}_{2}$. Linear Algebra Appl. 418 (2006), no. $1,85-111$.

[10] Draper, C. AND Martín, C.: Fine gradings on some exceptional algebras. In From Lie Algebras to Quantum Groups, 79-91. Centro Internacional de Matemática, 2007.

[11] Draper, C. and Martín, C.: Gradings on the Albert algebra and on $\mathfrak{f}_{4}$. Rev. Mat. Iberoam. 25 (2009), no. 3, 841-908.

[12] Draper, C., Martín, C. And Viruel, A.: Fine gradings on the Lie algebra $\mathfrak{d}_{4}$. Forum Math. 22 (2010), no. 5, 863-877.

[13] Draper, C. And Viruel, A.: Group Gradings on $\mathfrak{o}(8, \mathbb{C})$. Rep. Math. Phys. 61 (2008), no. 2, 265-280.

[14] Elduque, A.: Gradings on symmetric composition algebras. J. Algebra 322 (2009), no. $10,3542-3579$.

[15] Elduque, A.: Fine gradings on simple classical Lie algebras. J. Algebra 324 (2010), no. $12,3532-3571$.

[16] Griess, R.: Elementary abelian $p$-subgroups of algebraic groups. Geom. Dedicata 39 (1991), no. 3, 253-305.

[17] Havlíček, M., Patera, J. and Pelantová, E.: On Lie gradings. II. Linear Algebra Appl. 277 (1998), no. 1-3, 97-125.

[18] Havlíček, M., Patera, J. and Pelantová, E.: Fine gradings of the real forms of sl(3, C). Phys. Atomic Nuclei 61 (1998), no. 12, 2183-2186. 
[19] Jacobson, N.: Some groups of transformations defined by Jordan algebras. II. Groups of type $F_{4}$. J. Reine Angew. Math. 204 (1960), 74-98.

[20] Jackowski, S., McClure, J. And Oliver, B.: Homotopy classification of selfmaps of $B G$ via $G$-actions. II. Ann. of Math. (2) 135 (1992), no. 2, 227-270.

[21] Jackowski, S., McClure, J. and Oliver, B.: Maps between classifying spaces revisited. In The Čech centennial (Boston, MA, 1993), 263-298. Contemp. Math. 181, Amer. Math. Soc., Providence, RI, 1995.

[22] KAC, V. G.: Automorphisms of finite order of semisimple Lie algebras. Funct. Anal. Appl. 3 (1969), 252-254.

[23] Knus, M. A.: Quadratic and hermitian forms over rings. Grundlehren der Mathematischen Wissenschaften 294, Springer, Berlin, 1991.

[24] Knus, M. A., Merkurjev, A., Rost, M. and Tignol, J. P.: The book of involutions. American Mathematical Society Colloquium Publications 44, American Mathematical Society, Providence, RI, 1998.

[25] Martín, C.: Web page http://agt2.cie.uma.es/f4bis.zip.

[26] Platonov, V.P.: The theory of algebraic linear groups and periodic groups. Izv. Akad. Nauk SSSR Ser. Mat. 30 (1966), 573-620. English translation in Amer. Math. Soc. Transl. Ser. 269 (1968), 61-110.

[27] Viruel, A.: Mod 3 homotopy uniqueness of BF. J. Math. Kyoto Univ. 41 (2001), no. 4, 769-793.

Received April 9, 2010.

Cristina Draper Fontanals: Departamento de Matemática Aplicada, Escuela de las Ingenierías, Ampliación del Campus de Teatinos, Universidad de Málaga, 29071

Málaga, Spain.

E-mail: cdf@uma.es

Supported by the Spanish MCYT projects MTM2007-60333 and by the Junta de Andalucía PAI projects FQM-336, FQM-1215 and FQM-2467. 